\title{
Review Article \\ Brief Overview of a Decade of Genome-Wide Association Studies on Primary Hypertension
}

\author{
Afifah Binti Azam (D) and Elena Aisha Binti Azizan \\ Department of Medicine, The National University of Malaysia Medical Centre, Kuala Lumpur, Malaysia \\ Correspondence should be addressed to Elena Aisha Binti Azizan; elena.azizan@ukm.edu.my
}

Received 28 October 2017; Accepted 12 December 2017; Published 30 January 2018

Academic Editor: Raffaele Pezzani

Copyright ( 2018 Afifah Binti Azam and Elena Aisha Binti Azizan. This is an open access article distributed under the Creative Commons Attribution License, which permits unrestricted use, distribution, and reproduction in any medium, provided the original work is properly cited.

\begin{abstract}
Primary hypertension is widely believed to be a complex polygenic disorder with the manifestation influenced by the interactions of genomic and environmental factors making identification of susceptibility genes a major challenge. With major advancement in high-throughput genotyping technology, genome-wide association study (GWAS) has become a powerful tool for researchers studying genetically complex diseases. GWASs work through revealing links between DNA sequence variation and a disease or trait with biomedical importance. The human genome is a very long DNA sequence which consists of billions of nucleotides arranged in a unique way. A single base-pair change in the DNA sequence is known as a single nucleotide polymorphism (SNP). With the help of modern genotyping techniques such as chip-based genotyping arrays, thousands of SNPs can be genotyped easily. Large-scale GWASs, in which more than half a million of common SNPs are genotyped and analyzed for disease association in hundreds of thousands of cases and controls, have been broadly successful in identifying SNPs associated with heart diseases, diabetes, autoimmune diseases, and psychiatric disorders. It is however still debatable whether GWAS is the best approach for hypertension. The following is a brief overview on the outcomes of a decade of GWASs on primary hypertension.
\end{abstract}

\section{Introduction}

Hypertension is highly prevalent globally. The estimated number of people with uncontrolled hypertension is nearly 1 billion (around 15\% of the world population), with the number predicted to increase to 1.56 billion by the year 2025 [1]. Due to its high prevalence, hypertension is the leading risk factor for cardiovascular disease, stroke, and endstage kidney diseases. The increased risk of cardiovascular mortality and morbidity has led to the estimation that hypertension causes $13 \%$ of all deaths (around 7.5 million deaths worldwide) [2]. Patients are considered to have hypertension when their systolic blood pressure is $\geq 140 \mathrm{mmHg}$ and/or their diastolic blood pressure is $\geq 90 \mathrm{mmHg}$ [3]. However, raised blood pressure, even within the normal range, is positively and continuously related to mortality and morbidity-each increment of 20 (systolic)/10 (diastolic) $\mathrm{mmHg}$ of blood pressure doubles the risk of cardiovascular diseases
[2]. Hence, the number of people at risk is higher as the prevalence of raised blood pressure for adults (aged $\geq 25$ years) is around $40 \%$ [2].

The majority of hypertension in the general population occur idiopathically with no apparent causes and therefore are categorized as primary hypertension. The remaining hypertensive cases (about 5\%) are categorized as secondary hypertension as the raised blood pressure occur secondary to other causes/diseases, for example, hypertension due to aldosteronism, pheochromocytoma, renovascular diseases, or even Mendelian forms of hypertension [4, 5]. However, despite being classified as having no apparent cause, studies of familial aggregation on primary hypertensive patients have found associations of blood pressure among siblings and between parents and children, indicating that genetic factors contribute to the high blood pressure among primary hypertensive patients. Genetic factors have been estimated to explain $30-50 \%$ of the interindividual variation in blood 
pressure which significantly predisposes family (siblings/ children) of primary hypertensive patients to hypertension [6]. These heritable genetic factors, in addition to environment and demographic factors, play a major role in interindividual variation in blood pressure [7]. Therefore, extensive genetic research has been conducted over the years, including genome-wide association studies (GWASs), to help elucidate primary hypertension's heritability.

\section{Outcomes of Genome-Wide Association Studies on Primary Hypertension}

GWASs have identified over three hundred plus SNPs/loci associated with blood pressure and/or primary hypertension over the past decade (Table 1). Meta-analyses of GWASs have made the biggest contribution as they allowed for larger sample sizes and more extensive imputation panels. Despite these advancements, genetic variation identified so far only explains $\sim 3-6 \%$ of the variance for blood pressure, approximately $1 \mathrm{mmHg}$ per allele systolic blood pressure or $0.5 \mathrm{mmHg}$ per allele diastolic blood pressure [8-12]. Further, the vast majority of GWASs were performed predominantly in Caucasian populations with only a few studies assessing or replicating in other populations even though high blood pressure burden risk is ranked number one in Southeast Asia, Central Asia, North Africa, and Middle East [13-40]. This suggests the existence of many more undiscovered SNPs/loci or at the very least SNPs unique to other populations that are not of Caucasian ancestry. For example, one meta-analysis on Oriental populations found five Oriental-specific loci near CAPZA1, FIGN, ENPEP, NPR3, and PTPN11 (near C12orf51) associated with hypertension [22]. Either the differences in environmental exposures/lifestyle factors or genetic background can explain why ethnic/racial susceptibility loci exist. Nevertheless, as even a small increase in blood pressure can increase the risk of cardiovascular diseases, the biological pathways identified by these SNPs would still be useful in resolving many of the open questions regarding blood pressure pathophysiology.

\section{Biological Pathways Involved with Blood Pressure Pathophysiology}

Mendelian forms of hypertension and germline mutations causing early-onset hypertension have highlighted biological pathways that involve renal salt handling (WNK1, WNK4, KLHL3, and CUL3), ion transport (CACNA1D, CACNA1H, KCNJ5, SCNN1B, and SCNN1G), corticosteroidogenesis (CYP11B2, HSD11B2, NR3C2, CYP11B1, and CYP17A1), and vascular tone $(P D E 3 A)$ to regulate blood [41-44]. Thus, not surprisingly, GWASs have identified SNPs in or near to genes involved with these biological pathways associated with primary hypertension. In fact, one of the first few high-throughput genotyping was performed on only genes underlying monogenic hypertension and hypotension (not genome-wide) which found two renal sodium regulatory genes (KCNJ1 and NR3C2) to have SNPs associated with blood pressure in the general population [45].
3.1. Renal Salt Handling. One interesting SNP putatively involving renal salt-handling pathway was only linked to hypertension in an extreme case-control GWAS design [25]. This SNP, rs13333226, on chromosome 16 is in the $5^{\prime}$ region of $U M O D$ (combined $P$ value of $3.6 \times 10^{-11}$ ). The minor $\mathrm{G}$ allele of this SNP had an OR of 0.87 (95\% CI: 0.84-0.91) for hypertension, with the subject having the minor $\mathrm{G}$ allele having decreased urinary uromodulin and better renal function. The exclusive expression of uromodulin, the protein encoded by $U M O D$, in the thick portion of the ascending limb of Henle suggests that the SNP exerts its effect through sodium homeostasis [25]. Also based on renal expression, SNPs in or near to PAPPA2 and ADAMTS7 (rs61823001 and rs62011052, resp., [8]) are expected to play a role in the renal salt-handling pathway. Interestingly in regard to the protein encoded by ADAMTS7, angiotensin II stimulation induced renal expression of the protein [46]. Similarly, renal cortex expression of PAPPA2 in Dahl salt-sensitive rats responded to changes of salt diet supporting a role of the SNP in the renal salthanding pathway [47]. SNPs in FAM186B and ARHGAP24 on the other hand are postulated to play a role in renal function based on involvement with kidney diseases. Combining whole exome sequencing and homozygosity mapping in consanguineous families, FAM $186 B$ was identified as a novel candidate gene for monogenic, recessive nephronophthisis-related ciliopathies [48]. ARHGAP24 on the other hand is thought to play a role in renal cell carcinoma and focal segmental glomerulosclerosis most likely through RhoA and Racl signaling pathways [49, 50].

3.2. Ion Transport. Several SNPs in genes involved with ion transport have been associated with blood pressure (e.g., ATP2B1, CACNA1D, CACNA2D2, CACNB2, KCNK3, SLC4A7, and SLC39A8; Table 1). Of these, the one most studied and replicated are SNPs in ATP2B1 [9, 18, 22, 51]. Confirming the role of $A T P 2 B 1$ in blood pressure regulation is the vascular smooth muscle cell-specific knockout of $A T P 2 B 1$ mice which had higher systolic blood pressure and significantly increased phenylephrine-induced vasoconstrictions [52]. Similarly, silencing of ATP2B1 through injection of an SiRNA complex into mouse tail veins led to an increase in blood pressure and an increase in contractile response to phenylephrine [53]. These results support that ATP2B1 genetic variants are the causative gene for the association with blood pressure seen in GWASs. The other gene encoding an ion channel with significant supporting evidence is CACNA1D. This is because gain of function mutations in CACNA1D have been found to be causal for primary aldosteronism and for aldosterone-producing cell clusters [42, 54, 55]. As aldosterone is a key regulator of blood pressure, even small changes which may not pass the clinical threshold for primary aldosteronism may be causal for increase in blood pressure. Elevation of aldosterone may also be the mechanism of action for the other ion channels associated with primary hypertension as mutations in the ATPase $\mathrm{Na}^{+} / \mathrm{K}^{+}$transporting subunit alpha 1 and $\mathrm{G}$ proteinactivated inward rectifier $\mathrm{K}^{+}$channel 4 have also been found causal for primary aldosteronism and aldosterone-producing cell clusters $[55,56]$. 
TABLE 1: Loci associated with blood pressure and/or hypertension that have been identified through large-scale studies in the past decade.

\begin{tabular}{|c|c|c|c|c|c|c|c|c|}
\hline Locus name & SNP & $\begin{array}{l}\text { Chr } \\
\text { ID }\end{array}$ & $\begin{array}{c}\text { Chr } \\
\text { position }\end{array}$ & $\begin{array}{l}\text { Coded } \\
\text { allele }\end{array}$ & $\begin{array}{l}\text { Best } \\
\text { trait }\end{array}$ & $\begin{array}{l}\text { Effect size of best } \\
\text { trait (OR beta) }\end{array}$ & $\begin{array}{l}\text { Coded allele } \\
\text { frequency }\end{array}$ & Reporting article \\
\hline $2 q 36.3$ & rs2972146 & 2 & $226,235,982$ & $\mathrm{~T}$ & DBP & 0.17 & 0.19 & Surendran et al. [29] \\
\hline $7 q 32.1$ & rs4728142 & 7 & $128,933,913$ & A & SBP & -0.224 & 0.29 & Surendran et al. [29] \\
\hline$A B H D 17 C$ & rs35199222 & 15 & $80,720,696$ & $\mathrm{~A}$ & SBP & 0.322 & 0.18 & $\begin{array}{l}\text { Hoffmann et al. [8], } \\
\text { Warren et al. [26] }\end{array}$ \\
\hline$A B H D 17 C$ & rs11634851 & 15 & $80,736,624$ & G & SBP & 0.316 & 0.461 & Wain et al. [27] \\
\hline ABLIM3-SH3TC2 & rs9687065 & 5 & $149,011,577$ & A & DBP & 0.26 & 0.16 & Kato et al. [20] \\
\hline$A C E$ & rs4308 & 17 & $63,482,264$ & $\mathrm{~A}$ & DBP & 0.213 & 0.24 & $\begin{array}{l}\text { Hoffmann et al. [8], } \\
\text { Warren et al. [26] }\end{array}$ \\
\hline$A C O X 1$ & rs2467099 & 17 & $75,952,964$ & $\mathrm{~T}$ & SBP & -0.307 & 0.18 & $\begin{array}{l}\text { Hoffmann et al. [8], } \\
\text { Warren et al. [26] }\end{array}$ \\
\hline ADAMTS7-MORF4L1 & rs62012628 & 15 & $78,777,658$ & $\mathrm{~T}$ & DBP & -0.238 & 0.34 & $\begin{array}{l}\text { Hoffmann et al. [8], } \\
\text { Warren et al. [26] }\end{array}$ \\
\hline ADAMTS7-MORF4L1 & rs62011052 & 15 & $79,156,983$ & $\mathrm{C}$ & $\mathrm{PP}$ & -0.28 & 0.14 & Hoffmann et al. [8] \\
\hline ADAMTS8 & rs11222084 & 11 & $130,403,335$ & $\mathrm{~T}$ & $\mathrm{PP}$ & 0.337 & 0.21 & Wain et al. [19] \\
\hline ADAMTS9 & rs918466 & 3 & $64,724,577$ & $\mathrm{~A}$ & DBP & -0.204 & 0.35 & Ehret et al. [12] \\
\hline$A D C Y 3$ & rs55701159 & 2 & $24,916,727$ & $\mathrm{~T}$ & DBP & 0.285 & 0.1 & Warren et al. [26] \\
\hline$A D M$ & rs360157 & 11 & $9,732,674$ & $\mathrm{~T}$ & SBP & 0.413 & 0.44 & Ehret et al. [12] \\
\hline$A D M$ & rs7129220 & 11 & $10,350,538$ & $\mathrm{~A}$ & SBP & -0.619 & 0.058 & Ehret et al. [18] \\
\hline$A D M$ & rs7129220 & 11 & $10,350,538$ & $\mathrm{~A}$ & DBP & -0.299 & 0.058 & Ehret et al. [18] \\
\hline$A D O$ & rs10995311 & 10 & $62,805,174$ & G & DPB & -0.20 & 0.38 & $\begin{array}{l}\text { Liu et al. [23], } \\
\text { Surendran et al. [29] }\end{array}$ \\
\hline$A D R B 1$ & rs2782980 & 10 & $114,021,768$ & $\mathrm{~T}$ & $\mathrm{PP}$ & -0.338 & 0.28 & Wain et al. [19] \\
\hline ADRB1-RNU6-709P & rs10787517 & 10 & $114,055,047$ & A & SBP & 0.442 & 0.616 & Wain et al. [27] \\
\hline$A G T$ & rs2004776 & 1 & $230,712,956$ & $\mathrm{~T}$ & SBP & 0.42 & 0.41 & Johnson et al. [30] \\
\hline AKT2 & rs9710247 & 19 & $40,254,542$ & G & DBP & 0.252 & 0.44 & Wain et al. [27] \\
\hline$A M H-S F 3 A 2$ & rs740406 & 19 & $2,232,222$ & A & $\mathrm{PP}$ & -0.55 & 0.21 & Kato et al. [20] \\
\hline ARHGAP12 & rs 10826995 & 10 & $31,793,730$ & $\mathrm{~T}$ & $\mathrm{PP}$ & -0.212 & 0.3 & $\begin{array}{l}\text { Hoffmann et al. [8], } \\
\text { Warren et al. [26] }\end{array}$ \\
\hline ARHGAP24 & rs2014912 & 4 & $85,794,517$ & $\mathrm{~T}$ & SBP & 0.62 & 0.19 & Kato et al. [20] \\
\hline$A R N T L$ & rs900145 & 11 & $13,272,358$ & G & DBP & -0.25 & 0.43 & Liu et al. [23] \\
\hline$A R V C F$ & rs12628032 & 22 & $19,980,457$ & $\mathrm{~T}$ & $\mathrm{PP}$ & 0.24 & 0.27 & $\begin{array}{l}\text { Hoffmann et al. [8], } \\
\text { Warren et al. [26] }\end{array}$ \\
\hline$A R V C F$ & rs4819852 & 22 & $20,000,644$ & $\mathrm{~A}$ & $\mathrm{PP}$ & 0.261 & 0.29 & Wain et al. [27] \\
\hline$A T P 2 B 1$ & rs2681472 & 12 & $89,615,182$ & $\mathrm{~A}$ & DBP & 0.5 & 0.83 & Levy et al. [9] \\
\hline$A T P 2 B 1$ & rs2681492 & 12 & $89,619,312$ & $\mathrm{~T}$ & SBP & 1.26 & 0.21 & Levy et al. [9] \\
\hline$A T P 2 B 1$ & rs17249754 & 12 & $89,666,809$ & A & $\mathrm{BP}$ & 0.8 & 0.35 & Kelly et al. [31] \\
\hline BAT2-BAT5 & rs805303 & 6 & $31,648,589$ & G & SBP & 0.376 & 0.44 & Johnson et al. [30] \\
\hline$B D N F$ & rs11030119 & 11 & $27,706,555$ & $\mathrm{~A}$ & DBP & -0.163 & 0.26 & $\begin{array}{l}\text { Hoffmann et al. [8], } \\
\text { Warren et al. [26] }\end{array}$ \\
\hline BLK-GATA4 & rs2898290 & 8 & $11,576,400$ & $\mathrm{C}$ & SBP & NA & 0.38 & Ho et al. [33] \\
\hline C10orf107 & rs4590817 & 10 & $61,707,795$ & $\mathrm{C}$ & DBP & 0.436 & 0.16 & Wain et al. [27] \\
\hline C10orf107 & rs1530440 & 10 & $61,764,833$ & $\mathrm{~T}$ & DBP & 0.19 & 0.15 & Newton-Cheh et al. [10] \\
\hline $\begin{array}{l}\text { C10orf32, } \\
\text { C10orf32-ASMT }\end{array}$ & rs4409766 & 10 & $102,856,906$ & $\mathrm{~T}$ & SBP & 1.24 & 0.71 & Lu et al. [51] \\
\hline C17orf82-TBX2 & rs2240736 & 17 & $61,408,032$ & $\mathrm{~T}$ & MAP & 0.35 & 0.35 & Kato et al. [20] \\
\hline C20orf187 & rs1887320 & 20 & $10,985,350$ & A & SBP & 0.78 & 0.53 & Lu et al. [51] \\
\hline C2orf43 & rs2289081 & 2 & $20,682,080$ & $\mathrm{C}$ & $\mathrm{PP}$ & -0.223 & 0.31 & $\begin{array}{l}\text { Hoffmann et al. [8], } \\
\text { Warren et al. [26] }\end{array}$ \\
\hline C5orf56 & rs2188962 & 5 & $132,435,113$ & $\mathrm{~T}$ & DBP & -0.2 & 0.14 & $\begin{array}{l}\text { Liu et al. [23], } \\
\text { Surendran et al. }\end{array}$ \\
\hline
\end{tabular}


TABle 1: Continued.

\begin{tabular}{|c|c|c|c|c|c|c|c|c|}
\hline Locus name & SNP & $\begin{array}{l}\text { Chr } \\
\text { ID }\end{array}$ & $\begin{array}{c}\mathrm{Chr} \\
\text { position }\end{array}$ & $\begin{array}{l}\text { Coded } \\
\text { allele }\end{array}$ & $\begin{array}{l}\text { Best } \\
\text { trait }\end{array}$ & $\begin{array}{c}\text { Effect size of best } \\
\text { trait (OR beta) }\end{array}$ & $\begin{array}{l}\text { Coded allele } \\
\text { frequency }\end{array}$ & Reporting article \\
\hline CACNA1D & rs9810888 & 3 & $53,601,568$ & G & DBP & 0.39 & 0.39 & Lu et al. [51] \\
\hline CACNA2D2 & rs743757 & 3 & $50,438,947$ & $\mathrm{C}$ & DBP & 0.245 & 0.36 & $\begin{array}{l}\text { Hoffmann et al. [8], } \\
\text { Warren et al. [26] }\end{array}$ \\
\hline CACNB2 & rs1813353 & 10 & $18,418,519$ & $\mathrm{C}$ & DBP & 0.332 & 0.34 & Wain et al. [27] \\
\hline CACNB2 & rs11014166 & 10 & $18,419,869$ & A & DBP & 0.46 & 0.21 & Levy et al. [9] \\
\hline CAMKV-ACTBP 13 & rs36022378 & 3 & $49,876,272$ & $\mathrm{~T}$ & DBP & -0.202 & 0.11 & $\begin{array}{l}\text { Hoffmann et al. [8], } \\
\text { Warren et al. [26] }\end{array}$ \\
\hline CAPZA1 & rs10745332 & 1 & $112,646,431$ & $\mathrm{~A}$ & SBP & 0.96 & 0.82 & Lu et al. [51] \\
\hline CASC15 & rs6911827 & 6 & $22,130,372$ & $\mathrm{~T}$ & SBP & 0.296 & 0.30 & $\begin{array}{l}\text { Hoffmann et al. [8], } \\
\text { Warren et al. [26] }\end{array}$ \\
\hline CASZ1 & rs880315 & 1 & $10,736,809$ & $\mathrm{~T}$ & SBP & -0.475 & 0.39 & Ehret et al. [12] \\
\hline CCDC141 & rs79146658 & 2 & $178,921,341$ & $\mathrm{~T}$ & DBP & -0.311 & 0.03 & $\begin{array}{l}\text { Hoffmann et al. [8], } \\
\text { Warren et al. [26] }\end{array}$ \\
\hline $\begin{array}{l}\text { CCDC41-CEP83- } \\
\text { RN7SL } 483 P\end{array}$ & rs139236208 & 12 & $94,486,966$ & A & PP & -0.363 & 0.04 & $\begin{array}{l}\text { Hoffmann et al. [8], } \\
\text { Warren et al. [26] }\end{array}$ \\
\hline CCNE1 & rs62104477 & 19 & $29,804,084$ & $\mathrm{~T}$ & DBP & 0.177 & 0.19 & $\begin{array}{l}\text { Hoffmann et al. [8], } \\
\text { Warren et al. [26] }\end{array}$ \\
\hline CD34 & rs12731740 & 1 & $207,851,475$ & $\mathrm{~T}$ & PP & -0.249 & 0.08 & Warren et al. [26] \\
\hline$C D C 42 B P A$ & rs10916082 & 1 & $227,064,925$ & $\mathrm{~A}$ & DBP & -0.177 & 0.27 & Warren et al. [26] \\
\hline $\mathrm{CDH} 13$ & rs7500448 & 16 & $83,012,185$ & A & PP & 0.329 & 0.17 & $\begin{array}{l}\text { Hoffmann et al. [8], } \\
\text { Warren et al. [26] }\end{array}$ \\
\hline $\mathrm{CDH} 17$ & rs2446849 & 8 & $94,091,269$ & $\mathrm{~T}$ & SBP & -0.63 & 0.22 & Zhu et al. [32] \\
\hline CELA2A & rs 1042010 & 1 & $15,467,418$ & A & SBP & 0.412 & 0.19 & $\begin{array}{c}\text { Hoffmann et al. [8], } \\
\text { Warren et al. [26] }\end{array}$ \\
\hline CELA2A & rs3820068 & 1 & $15,471,702$ & A & SBP & 0.425 & 0.19 & Wain et al. [27] \\
\hline CEP164 & rs8258 & 11 & $117,412,960$ & $\mathrm{~T}$ & PP & 0.236 & 0.47 & $\begin{array}{l}\text { Hoffmann et al. [8], } \\
\text { Warren et al. [26] }\end{array}$ \\
\hline CEP68 & rs74181299 & 2 & $65,056,838$ & $\mathrm{~T}$ & $\mathrm{PP}$ & 0.23 & 0.46 & $\begin{array}{l}\text { Hoffmann et al. [8], } \\
\text { Warren et al. [26] }\end{array}$ \\
\hline CERS5 & rs7302981 & 12 & $50,144,032$ & A & DBP & 0.249 & 0.30 & $\begin{array}{l}\text { Liu et al. [23], } \\
\text { Surendran et al. [29] }\end{array}$ \\
\hline CFDP1 & rs11643209 & 16 & $75,297,146$ & $\mathrm{~T}$ & SBP & -0.339 & 0.47 & $\begin{array}{l}\text { Hoffmann et al. [8], } \\
\text { Warren et al. [26] }\end{array}$ \\
\hline CHIC2 & rs871606 & 4 & $53,933,078$ & $\mathrm{~T}$ & $\mathrm{PP}$ & 0.429 & 0.21 & Wain et al. [19] \\
\hline chr15mb95 & rs12906962 & 15 & $94,768,842$ & $\mathrm{~T}$ & DBP & -0.221 & 0.42 & $\begin{array}{l}\text { Hoffmann et al. [8], } \\
\text { Warren et al. [26] }\end{array}$ \\
\hline chr1mb25 & rs6686889 & 1 & $24,703,979$ & $\mathrm{~T}$ & DBP & 0.185 & 0.37 & Warren et al. [26] \\
\hline chr1mb9 & rs9662255 & 1 & $9,381,890$ & A & $\mathrm{PP}$ & -0.207 & 0.41 & $\begin{array}{l}\text { Hoffmann et al. [8], } \\
\text { Warren et al. [26] }\end{array}$ \\
\hline CHST12-LFNG & rs2969070 & 7 & $2,472,910$ & $\mathrm{~A}$ & DBP & -0.205 & 0.21 & Ehret et al. [12] \\
\hline CMIP & rs8059962 & 16 & $81,540,592$ & $\mathrm{~T}$ & DBP & -0.170 & 0.45 & Warren et al. [26] \\
\hline CNNM2 & rs11191548 & 10 & $103,086,421$ & $\mathrm{C}$ & SBP & 1.082 & 0.09 & Wain et al. [27] \\
\hline COL21A1 & rs1925153 & 6 & $56,237,982$ & $\mathrm{~T}$ & $\mathrm{PP}$ & -0.21 & 0.44 & Liu et al. [23] \\
\hline CPEB4 & rs72812846 & 5 & $173,950,633$ & A & DBP & -0.209 & 0.11 & $\begin{array}{c}\text { Hoffmann et al. [8], } \\
\text { Warren et al. [26] }\end{array}$ \\
\hline$C R A C R 2 B$ & rs7126805 & 11 & 828,916 & A & $\mathrm{PP}$ & 0.222 & $<0.01$ & Warren et al. [26] \\
\hline$C R K$ & rs12941318 & 17 & $1,430,304$ & $\mathrm{~T}$ & SBP & -0.269 & 0.37 & $\begin{array}{l}\text { Hoffmann et al. [8], } \\
\text { Warren et al. [26] }\end{array}$ \\
\hline CRYAA-SIK1-RRP1B & rs12627651 & 21 & $43,340,723$ & A & SBP & 0.503 & 0.19 & $\begin{array}{l}\text { Ehret et al. [12], } \\
\text { Surendran et al. [29] }\end{array}$ \\
\hline
\end{tabular}


TABle 1: Continued.

\begin{tabular}{|c|c|c|c|c|c|c|c|c|}
\hline Locus name & SNP & $\begin{array}{l}\text { Chr } \\
\text { ID }\end{array}$ & $\begin{array}{c}\mathrm{Chr} \\
\text { position }\end{array}$ & $\begin{array}{l}\text { Coded } \\
\text { allele }\end{array}$ & $\begin{array}{l}\text { Best } \\
\text { trait }\end{array}$ & $\begin{array}{l}\text { Effect size of best } \\
\text { trait (OR beta) }\end{array}$ & $\begin{array}{l}\text { Coded allele } \\
\text { frequency }\end{array}$ & Reporting article \\
\hline CSK & rs1378942 & 15 & $74,785,026$ & $\mathrm{~A}$ & DBP & 0.371 & 0.65 & Wain et al. [27] \\
\hline CYB561-LOC342541 & rs4459609 & 17 & $63,471,587$ & $\mathrm{~A}$ & DBP & 0.198 & 0.61 & Wain et al. [27] \\
\hline CYP17A1-NT5C2 & rs 1004467 & 10 & $102,834,750$ & A & SBP & 1.2 & 0.16 & $\begin{array}{l}\text { Levy et al. [9], } \\
\text { Newton-Cheh et al. [10] }\end{array}$ \\
\hline CYP1A1-ULK3 & rs6495122 & 15 & $74,833,304$ & A & DBP & 0.45 & 0.29 & $\begin{array}{l}\text { Levy et al. [9], } \\
\text { Newton-Cheh et al. [10] }\end{array}$ \\
\hline CYP2C19 & rs4494250 & 10 & $94,804,000$ & $\mathrm{~A}$ & $\mathrm{DPB}$ & 0.21 & 0.22 & Liu et al. [23] \\
\hline $\mathrm{DBH}$ & rs6271 & 9 & $133,657,152$ & $\mathrm{~T}$ & DBP & -0.423 & 0.04 & Ehret et al. [12] \\
\hline DNM3 & rs12405515 & 1 & $172,388,301$ & $\mathrm{~T}$ & DBP & -0.165 & 0.47 & $\begin{array}{l}\text { Hoffmann et al. [8], } \\
\text { Warren et al. [26] }\end{array}$ \\
\hline$D P E P 1$ & rs1126464 & 16 & $89,637,957$ & $\mathrm{C}$ & DBP & 0.275 & 0.26 & $\begin{array}{l}\text { Liu et al. [23], } \\
\text { Surendran et al. [29] }\end{array}$ \\
\hline$E B F 1$ & rs11953630 & 5 & $158,418,394$ & $\mathrm{~T}$ & DBP & -0.281 & 0.18 & Johnson et al. [30] \\
\hline EBF2 & rs6557876 & 8 & $26,043,159$ & $\mathrm{~T}$ & SBP & -0.411 & 0.33 & Wain et al. [27] \\
\hline ENPEP & rs6825911 & 4 & $110,460,482$ & $\mathrm{C}$ & DBP & 0.39 & 0.42 & Kato et al. [22] \\
\hline ESR1 & rs 13192976 & 6 & $151,991,280$ & A & PP & -0.332 & 0.21 & $\begin{array}{l}\text { Hoffmann et al. [8], } \\
\text { Warren et al. [26] }\end{array}$ \\
\hline$F A F 1$ & rs147696085 & 1 & $50,556,195$ & G & $\mathrm{PP}$ & 0.298 & 0.06 & Hoffmann et al. [8] \\
\hline FAM186B & rs7977389 & 12 & $49,587,939$ & $\mathrm{~T}$ & $\mathrm{PP}$ & 0.237 & 0.18 & Hoffmann et al. [8] \\
\hline FAM208A & rs9827472 & 3 & $56,692,618$ & $\mathrm{~T}$ & DBP & -0.177 & 0.46 & $\begin{array}{l}\text { Hoffmann et al. [8], } \\
\text { Warren et al. [26] }\end{array}$ \\
\hline FBLN5 & rs2244643 & 14 & $91,892,678$ & A & $\mathrm{PP}$ & -0.213 & 0.29 & Hoffmann et al. [8] \\
\hline FBN2 & rs6595838 & 5 & $128,532,506$ & $\mathrm{~A}$ & SBP & 0.344 & 0.41 & $\begin{array}{l}\text { Hoffmann et al. [8], } \\
\text { Warren et al. [26] }\end{array}$ \\
\hline FBXL19 & rs72799341 & 16 & $30,925,422$ & A & DBP & 0.185 & 0.27 & $\begin{array}{l}\text { Hoffmann et al. [8], } \\
\text { Warren et al. [26] }\end{array}$ \\
\hline FER1L5 & rs7599598 & 2 & $96,686,103$ & A & DBP & -0.31 & 0.42 & Ganesh et al. [34] \\
\hline FERMT2 & rs9888615 & 14 & $52,910,822$ & $\mathrm{~T}$ & SBP & -0.318 & 0.36 & $\begin{array}{l}\text { Hoffmann et al. [8], } \\
\text { Warren et al. [26] }\end{array}$ \\
\hline FGD5 & rs11128722 & 3 & $14,916,619$ & A & SBP & -0.383 & 0.41 & Ehret et al. [12] \\
\hline FGF5 & rs16998073 & 4 & $80,263,187$ & $\mathrm{~T}$ & DBP & 0.21 & 0.23 & Newton-Cheh et al. [10] \\
\hline FGGY-HSD52 & rs3889199 & 1 & $59,188,070$ & A & PP & 0.351 & 0.14 & $\begin{array}{l}\text { Hoffmann et al. [8], } \\
\text { Warren et al. [26] }\end{array}$ \\
\hline FIGN-PRPS1P1 & rs16849211 & 2 & $164,043,173$ & $\mathrm{~T}$ & $\mathrm{PP}$ & 0.364 & 0.23 & Wain et al. [27] \\
\hline FIGN-PRPS1P1 & rs 1446468 & 2 & $164,106,976$ & $\mathrm{C}$ & SBP & 0.538 & 0.55 & Wain et al. [27] \\
\hline FIGN-GRB14 & rs16849225 & 2 & $164,050,310$ & $\mathrm{C}$ & SBP & 0.75 & 0.23 & $\begin{array}{c}\text { Ehret et al. [18], } \\
\text { Kato et al. } \\
\text { [22], Wain et al. [19] }\end{array}$ \\
\hline FLJ32810-TMEM133 & rs633185 & 11 & $100,722,807$ & G & SBP & -0.565 & 0.36 & Johnson et al. [30] \\
\hline FN1 & rs 1250259 & 2 & $215,435,759$ & A & $\mathrm{PP}$ & -0.314 & 0.23 & $\begin{array}{l}\text { Hoffmann et al. [8], } \\
\text { Warren et al. [26] }\end{array}$ \\
\hline FNDC1 & rs449789 & 6 & $159,278,093$ & $\mathrm{C}$ & PP & 0.359 & 0.15 & $\begin{array}{l}\text { Hoffmann et al. [8], } \\
\text { Warren et al. [26] }\end{array}$ \\
\hline FOSL2 & rs7562 & 2 & $28,412,873$ & $\mathrm{~T}$ & SBP & 0.263 & 0.50 & Warren et al. [26] \\
\hline FRMD3 & rs115795127 & 9 & $83,378,986$ & $\mathrm{~T}$ & $\mathrm{BP}$ & NA & NR & Liang et al. [35] \\
\hline FURIN-FES & rs2521501 & 15 & $90,894,158$ & $\mathrm{~T}$ & SBP & 0.65 & 0.21 & Johnson et al. [30] \\
\hline GATA2 & rs62270945 & 3 & $128,483,046$ & $\mathrm{~T}$ & $\mathrm{PP}$ & 0.607 & 0.01 & $\begin{array}{l}\text { Hoffmann et al. [8], } \\
\text { Warren et al. [26] }\end{array}$ \\
\hline GJA1 & rs11154027 & 6 & $121,460,244$ & $\mathrm{~T}$ & PP & 0.207 & 0.38 & Warren et al. [26] \\
\hline GNAS-EDN3 & rs6015450 & 20 & $59,176,062$ & G & SBP & 0.896 & 0.10 & Johnson et al. [30] \\
\hline GOSR2 & rs17608766 & 17 & $46,935,905$ & $\mathrm{~T}$ & SBP & -0.556 & 0.05 & Johnson et al. [30] \\
\hline
\end{tabular}


TABle 1: Continued.

\begin{tabular}{|c|c|c|c|c|c|c|c|c|}
\hline Locus name & SNP & $\begin{array}{l}\text { Chr } \\
\text { ID }\end{array}$ & $\begin{array}{c}\text { Chr } \\
\text { position }\end{array}$ & $\begin{array}{l}\text { Coded } \\
\text { allele }\end{array}$ & $\begin{array}{l}\text { Best } \\
\text { trait }\end{array}$ & $\begin{array}{l}\text { Effect size of best } \\
\text { trait (OR beta) }\end{array}$ & $\begin{array}{l}\text { Coded allele } \\
\text { frequency }\end{array}$ & Reporting article \\
\hline GPAT2-FAHD2CP & rs2579519 & 2 & $96,009,418$ & $\mathrm{~T}$ & $\mathrm{DBP}$ & -0.197 & 0.41 & Warren et al. [26] \\
\hline GPATCH2 & rs12408022 & 1 & $217,545,447$ & $\mathrm{~T}$ & DBP & 0.198 & 0.26 & $\begin{array}{l}\text { Hoffmann et al. [8], } \\
\text { Warren et al. [26] }\end{array}$ \\
\hline GPR20 & rs34591516 & 8 & $141,356,987$ & $\mathrm{~T}$ & SBP & 0.323 & 0.05 & Surendran et al. [29] \\
\hline GPR20 & rs78192203 & 8 & $141,364,973$ & $\mathrm{~T}$ & $\mathrm{BP}$ & NA & NR & Liang et al. [35] \\
\hline GPR98/ARRDC3 & rs10474346 & 5 & $91,268,322$ & $\mathrm{C}$ & DBP & 1.1 & 0.31 & Fox et al. [36] \\
\hline GTF2B & rs 10922502 & 1 & $88,894,475$ & A & SBP & -0.382 & 0.34 & $\begin{array}{l}\text { Hoffmann et al. [8], } \\
\text { Warren et al. [26] }\end{array}$ \\
\hline GUCY1A3 & rs13143871 & 4 & $155,698,052$ & $\mathrm{~T}$ & SBP & 0.96 & 0.80 & Lu et al. [51] \\
\hline GUCY1A3-GUCY1B3 & rs13139571 & 4 & $155,724,361$ & $\mathrm{C}$ & DBP & 0.26 & 0.21 & Johnson et al. [30] \\
\hline GYPA_HHIP & rs4292285 & 4 & $144,350,802$ & $\mathrm{~T}$ & DBP & 0.177 & 0.41 & Hoffmann et al. [8] \\
\hline $\begin{array}{l}\text { HAAO-RNU6-242P- } \\
\text { AC016735.1 }\end{array}$ & rs13403122 & 2 & $42,851,618$ & $\mathrm{C}$ & DBP & 0.226 & 0.20 & $\begin{array}{l}\text { Hoffmann et al. [8], } \\
\text { Warren et al. [26] }\end{array}$ \\
\hline HDAC9 & rs2107595 & 7 & $19,009,765$ & A & $\mathrm{PP}$ & 0.31 & 0.25 & Kato et al. [20] \\
\hline HFE & rs1799945 & 6 & $26,090,951$ & G & DBP & 0.457 & 0.09 & Johnson et al. [30] \\
\hline HFE & rs1800562 & 6 & $26,092,913$ & $\mathrm{~A}$ & DBP & 0.394 & 0.06 & Wain et al. [27] \\
\hline HIPK2 & rs1011018 & 7 & $139,763,465$ & $\mathrm{~A}$ & SBP & -0.329 & 0.35 & Warren et al. [26] \\
\hline HIVEP3 & rs7515635 & 1 & $41,942,399$ & $\mathrm{~T}$ & SBP & 0.336 & 0.47 & Ehret et al. [12] \\
\hline HM13-ID1 & rs6060114 & 20 & $31,581,870$ & $\mathrm{~T}$ & DBP & 0.267 & 0.27 & Hoffmann et al. [8] \\
\hline HNF4G-RNU2-54P & rs1449544 & 8 & $75,679,645$ & $\mathrm{~A}$ & $\mathrm{PP}$ & 0.183 & 0.41 & Hoffmann et al. [8] \\
\hline НОТТІР & rs1859168 & 7 & $27,202,740$ & $\mathrm{C}$ & DBP & 0.436 & 0.92 & Wain et al. [27] \\
\hline HOXA3 & rs6969780 & 7 & $27,119,517$ & $\mathrm{C}$ & $\mathrm{BP}$ & NA & NR & Liang et al. [35] \\
\hline HOXA-EVX1 & rs17428471 & 7 & $27,298,248$ & $\mathrm{~T}$ & SBP & 1.2 & 0.08 & Franceschini et al. [24] \\
\hline HOXB7 & rs7406910 & 17 & $48,610,894$ & $\mathrm{~T}$ & SBP & -0.456 & 0.12 & Surendran et al. [29] \\
\hline HRCT1 & rs76452347 & 9 & $35,906,474$ & $\mathrm{~T}$ & DBP & -0.25 & 0.15 & Liu et al. [23] \\
\hline HSD52-LOC105378756 & rs10889130 & 1 & $59,148,708$ & $\mathrm{~A}$ & $\mathrm{PP}$ & 0.288 & 0.33 & Wain et al. [27] \\
\hline HSPB7 & rs1048238 & 1 & $16,015,154$ & $\mathrm{~T}$ & SBP & 0.366 & 0.02 & Wain et al. [27] \\
\hline$I G F B P 3$ & rs11977526 & 7 & $45,968,511$ & A & DBP & 0.3 & 0.44 & $\begin{array}{l}\text { Zhu et al. [32], } \\
\text { Liu et al. [23] }\end{array}$ \\
\hline INPP5B & rs871524 & 1 & $37,945,773$ & A & $\mathrm{PP}$ & 0.228 & 0.33 & Wain et al. [27] \\
\hline INSR & rs7248104 & 19 & $7,224,420$ & A & $\mathrm{PP}$ & -0.20 & 0.35 & Liu et al. [23] \\
\hline INSR & rs36047283 & 19 & $7,255,690$ & G & SBP & 0.801 & 0.11 & Wain et al. [27] \\
\hline ITGA11 & rs1563894 & 15 & $68,343,437$ & $\mathrm{~A}$ & SBP & -0.093 & 0.18 & Parmar et al. [37] \\
\hline$J A G 1$ & rs1327235 & 20 & $10,988,382$ & G & DBP & 0.302 & 0.46 & Johnson et al. [30] \\
\hline JAG1-LOC101929395 & rs6040076 & 20 & $10,678,234$ & $\mathrm{C}$ & $\mathrm{PP}$ & 0.285 & 0.49 & Wain et al. [27] \\
\hline KCNH4-HSD17B1 & rs79089478 & 17 & $42,165,223$ & $\mathrm{~T}$ & $\mathrm{PP}$ & 0.584 & 0.01 & Warren et al. [26] \\
\hline KCNK3 & rs1275988 & 2 & $26,691,496$ & $\mathrm{~T}$ & SBP & -0.6 & 0.41 & Ganesh et al. [34] \\
\hline KIAA0753 & rs7226020 & 17 & $6,570,508$ & $\mathrm{~T}$ & $\mathrm{PP}$ & -0.256 & 0.38 & $\begin{array}{l}\text { Hoffmann et al. [8], } \\
\text { Warren et al. [26] }\end{array}$ \\
\hline KIAA1462 & rs9337951 & 10 & $30,028,144$ & A & $\mathrm{PP}$ & 0.28 & 0.26 & $\begin{array}{l}\text { Hoffmann et al. [8], } \\
\text { Warren et al. [26] }\end{array}$ \\
\hline L3MBTL4 & rs403814 & 18 & $6,282,594$ & A & $\mathrm{BP}$ & 1.15 & NR & Liu et al. [23] \\
\hline LHFPL2 & rs 10057188 & 5 & $78,541,966$ & A & $\mathrm{PP}$ & -0.205 & 0.24 & $\begin{array}{l}\text { Hoffmann et al. [8], } \\
\text { Warren et al. [26] }\end{array}$ \\
\hline LINC01615-THBS2 & rs1322639 & 6 & $169,187,008$ & A & $\mathrm{PP}$ & 0.316 & 0.33 & $\begin{array}{l}\text { Hoffmann et al. [8], } \\
\text { Warren et al. [26] }\end{array}$ \\
\hline$L M O 1$ & rs110419 & 11 & $8,231,306$ & A & $\mathrm{DBP}$ & 0.159 & 0.43 & Surendran et al. [29] \\
\hline LOC101928278 & rs10932679 & 2 & $216,787,868$ & $\mathrm{~T}$ & $\mathrm{PP}$ & 0.226 & 0.19 & Wain et al. [27] \\
\hline LOC102723446 & rs10260816 & 7 & $45,970,501$ & G & $\mathrm{PP}$ & 0.298 & 0.43 & Wain et al. [27] \\
\hline
\end{tabular}


TABle 1: Continued.

\begin{tabular}{|c|c|c|c|c|c|c|c|c|}
\hline Locus name & SNP & $\begin{array}{l}\text { Chr } \\
\text { ID }\end{array}$ & $\begin{array}{c}\text { Chr } \\
\text { position }\end{array}$ & $\begin{array}{l}\text { Coded } \\
\text { allele }\end{array}$ & $\begin{array}{l}\text { Best } \\
\text { trait }\end{array}$ & $\begin{array}{c}\text { Effect size of best } \\
\text { trait (OR beta) }\end{array}$ & $\begin{array}{l}\text { Coded allele } \\
\text { frequency }\end{array}$ & Reporting article \\
\hline $\begin{array}{l}\text { LOC105369687- } \\
\text { LOC105369688 }\end{array}$ & rs73075659 & 12 & $20,220,607$ & G & SBP & 0.357 & 0.31 & Wain et al. [27] \\
\hline LOC105370003 & rs11067763 & 12 & $115,760,536$ & A & DBP & 0.51 & 0.62 & Lu et al. [51] \\
\hline $\begin{array}{l}\text { LOC105371811- } \\
\text { LOC105371812 }\end{array}$ & rs79917357 & 17 & $48,747,312$ & A & SBP & 0.342 & 0.17 & Wain et al. [27] \\
\hline $\begin{array}{l}\text { LOC105374567- } \\
\text { LOC102723854 }\end{array}$ & rs72876037 & 2 & $42,967,456$ & $\mathrm{~T}$ & SBP & 0.534 & 0.12 & Wain et al. [27] \\
\hline LOC105379231 & rs9693857 & 8 & $9,409,607$ & $\mathrm{~T}$ & SBP & 0.337 & 0.45 & Wain et al. [27] \\
\hline $\begin{array}{l}\text { LOC107986913- } \\
\text { LOC105379224 }\end{array}$ & rs7826238 & 8 & $8,529,585$ & $\mathrm{~T}$ & SBP & 0.335 & 0.47 & Wain et al. [27] \\
\hline LOC283335 & rs73099903 & 12 & $53,046,995$ & $\mathrm{~T}$ & SBP & 0.768 & 0.06 & Wain et al. [27] \\
\hline LRP12/ZFPM2 & rs35783704 & 8 & $104,954,030$ & A & SBP & -0.609 & 0.03 & Wain et al. [27] \\
\hline LRRC10B-SYT7 & rs751984 & 11 & $61,510,774$ & $\mathrm{~T}$ & MAP & 0.33 & 0.27 & $\begin{array}{l}\text { Kato et al. [20], } \\
\text { Ehret et al. [12] }\end{array}$ \\
\hline LSP1-TNNT3 & rs661348 & 11 & $1,884,062$ & $\mathrm{~T}$ & MAP & -0.65 & 0.42 & Johnson et al. [30] \\
\hline MAP4 & rs319690 & 3 & $47,885,994$ & $\mathrm{~T}$ & DBP & 0.282 & 0.41 & Wain et al. [19] \\
\hline MAPK4-MRO & rs36010659 & 18 & $50,757,579$ & $\mathrm{~T}$ & PP & 0.25 & 0.12 & $\begin{array}{l}\text { Hoffmann et al. [8], } \\
\text { Warren et al. [26] }\end{array}$ \\
\hline$M C F 2 L$ & rs9549328 & 13 & $112,981,842$ & $\mathrm{~T}$ & SBP & 0.318 & 0.22 & $\begin{array}{l}\text { Hoffmann et al. [8], } \\
\text { Warren et al. [26] }\end{array}$ \\
\hline MECOM & rs419076 & 3 & $169,383,098$ & $\mathrm{~T}$ & SBP & 0.409 & 0.42 & Johnson et al. [30] \\
\hline METTL21A-AC079767.3 & rs55780018 & 2 & $207,661,416$ & $\mathrm{~T}$ & SBP & -0.391 & 0.35 & $\begin{array}{l}\text { Hoffmann et al. [8], } \\
\text { Warren et al. [26] }\end{array}$ \\
\hline MIR1263 & rs16833934 & 3 & $164,019,462$ & G & DBP & -1.63 & 0.31 & Simino et al. [38] \\
\hline MKLN1 & rs13238550 & 7 & $131,374,297$ & $\mathrm{~A}$ & SBP & 0.331 & 0.33 & Warren et al. [26] \\
\hline MOV10 & rs12129649 & 1 & $112,688,881$ & $\mathrm{~T}$ & DBP & 0.548 & 0.06 & Wain et al. [27] \\
\hline MRAS & rs2306374 & 3 & $138,401,110$ & $\mathrm{~T}$ & DBP & -0.184 & 0.08 & $\begin{array}{c}\text { Hoffmann et al. [8], } \\
\text { Warren et al. [26] }\end{array}$ \\
\hline$M R C 2$ & rs740698 & 17 & $62,689,790$ & $\mathrm{~T}$ & PP & -0.228 & 0.41 & Warren et al. [26] \\
\hline MSRA & rs11249992 & 8 & $10,362,902$ & A & SBP & 0.293 & 0.38 & Wain et al. [27] \\
\hline MTAP & rs4364717 & 9 & $21,801,531$ & A & DBP & -0.175 & 0.43 & Warren et al. [26] \\
\hline MTF1-SF3A3 & rs4360494 & 1 & $37,990,219$ & $\mathrm{C}$ & PP & 0.278 & 0.38 & $\begin{array}{l}\text { Hoffmann et al. [8], } \\
\text { Warren et al. [26] }\end{array}$ \\
\hline MTHFR & rs17367504 & 1 & $11,802,721$ & G & DBP & 0.526 & 0.15 & Wain et al. [27] \\
\hline MTHFR-NPPB & rs4846049 & 1 & $11,790,308$ & $\mathrm{~T}$ & DBP & -0.55 & 0.37 & Johnson et al. [30] \\
\hline MYEOV & rs67330701 & 11 & $69,312,240$ & $\mathrm{~T}$ & DBP & -0.367 & 0.12 & $\begin{array}{l}\text { Hoffmann et al. [8], } \\
\text { Warren et al. [26] }\end{array}$ \\
\hline MYH6 & rs452036 & 14 & $23,396,676$ & A & PP & -0.282 & 0.34 & $\begin{array}{l}\text { Liu et al. [23], } \\
\text { Surendran et al. [29] }\end{array}$ \\
\hline$N A D K-C P S F 3 L$ & rs139385870 & 1 & $1,754,504$ & $\mathrm{D}$ & SBP & -0.352 & 0.33 & $\begin{array}{l}\text { Hoffmann et al. [8], } \\
\text { Warren et al. [26] }\end{array}$ \\
\hline NFKBIA & rs8904 & 14 & $35,402,011$ & $\mathrm{~A}$ & SBP & 0.377 & 0.40 & Wain et al. [27] \\
\hline NME7 & rs7519279 & 1 & $169,238,123$ & G & $\mathrm{PP}$ & 0.218 & 0.13 & Hoffmann et al. [8] \\
\hline NOS3 & rs3918226 & 7 & $150,993,088$ & $\mathrm{~T}$ & DBP & 0.83 & 0.03 & Johnson et al. [30] \\
\hline $\mathrm{NOTCH} 3$ & rs10418305 & 19 & $15,167,997$ & $\mathrm{C}$ & $\mathrm{PP}$ & -0.282 & 0.13 & Hoffmann et al. [8] \\
\hline NOV & rs2071518 & 8 & $119,423,572$ & $\mathrm{~T}$ & $\mathrm{PP}$ & 0.312 & 0.32 & Wain et al. [19] \\
\hline NOX4 & rs2289125 & 11 & $89,491,285$ & A & PP & -0.377 & 0.32 & $\begin{array}{l}\text { Hoffmann et al. [8], } \\
\text { Warren et al. [26] }\end{array}$ \\
\hline NPNT & rs13112725 & 4 & $105,990,585$ & $\mathrm{C}$ & SBP & 0.435 & 0.34 & $\begin{array}{l}\text { Hoffmann et al. [8], } \\
\text { Warren et al. [26] }\end{array}$ \\
\hline$N P P A-A S 1, N P P A$ & rs12744757 & 1 & $11,846,764$ & $\mathrm{~T}$ & SBP & 0.695 & 0.06 & Wain et al. [27] \\
\hline
\end{tabular}


TABLe 1: Continued.

\begin{tabular}{|c|c|c|c|c|c|c|c|c|}
\hline Locus name & SNP & $\begin{array}{l}\text { Chr } \\
\text { ID }\end{array}$ & $\begin{array}{c}\mathrm{Chr} \\
\text { position }\end{array}$ & $\begin{array}{l}\text { Coded } \\
\text { allele }\end{array}$ & $\begin{array}{l}\text { Best } \\
\text { trait }\end{array}$ & $\begin{array}{c}\text { Effect size of best } \\
\text { trait (OR beta) }\end{array}$ & $\begin{array}{l}\text { Coded allele } \\
\text { frequency }\end{array}$ & Reporting article \\
\hline$N P R 1$ & rs35479618 & 1 & $153,689,947$ & A & SBP & 1.34 & 0.01 & Liu et al. [23] \\
\hline NPR3-C5orf23 & rs1173771 & 5 & $32,814,922$ & $\mathrm{C}$ & SBP & 0.63 & 0.34 & $\begin{array}{c}\text { Johnson et al. [30], } \\
\text { Kato et al. [22] }\end{array}$ \\
\hline OBFC1 & rs4387287 & 10 & $103,918,139$ & $\mathrm{~A}$ & SBP & 0.338 & 0.32 & Surendran et al. [29] \\
\hline OR5B12 & rs11229457 & 11 & $58,439,730$ & $\mathrm{~T}$ & SBP & -0.312 & 0.22 & Surendran et al. [29] \\
\hline OSR1 & rs 1344653 & 2 & $19,531,084$ & A & $\mathrm{PP}$ & -0.27 & 0.38 & Kato et al. [20] \\
\hline PABPC4 & rs4660293 & 1 & $39,562,508$ & G & DBP & 0.27 & 0.10 & Liu et al. [23] \\
\hline PALLD-chr $4 m b 174$ & rs1566497 & 4 & $168,795,997$ & $\mathrm{~A}$ & $\mathrm{PP}$ & 0.236 & 0.23 & $\begin{array}{l}\text { Hoffmann et al. [8], } \\
\text { Warren et al. [26] }\end{array}$ \\
\hline PAPPA2 & rs61823001 & 1 & $176,664,440$ & G & $\mathrm{PP}$ & 0.31 & 0.03 & Hoffmann et al. [8] \\
\hline$P A X 2$ & rs112184198 & 10 & $100,844,757$ & A & SBP & -0.659 & 0.05 & $\begin{array}{l}\text { Hoffmann et al. [8], } \\
\text { Warren et al. [26] }\end{array}$ \\
\hline PDE10A & rs147212971 & 6 & $165,764,963$ & $\mathrm{~T}$ & DBP & -0.360 & 0.13 & $\begin{array}{l}\text { Hoffmann et al. [8], } \\
\text { Warren et al. [26] }\end{array}$ \\
\hline$P D E 3 A$ & rs12579720 & 12 & $20,020,830$ & $\mathrm{C}$ & DBP & -0.32 & 0.46 & Kato et al. [20] \\
\hline PDE5A & rs66887589 & 4 & $119,588,124$ & $\mathrm{~T}$ & DBP & -0.215 & 0.50 & $\begin{array}{l}\text { Hoffmann et al. [8], } \\
\text { Warren et al. [26] }\end{array}$ \\
\hline PHACTR1 & rs9349379 & 6 & $12,903,725$ & A & SBP & 0.289 & 0.38 & Surendran et al. [29] \\
\hline PHIP & rs10943605 & 6 & $78,945,760$ & A & DBP & 0.18 & 0.49 & Liu et al. [23] \\
\hline PIK3CG & rs17477177 & 7 & $106,771,412$ & $\mathrm{~T}$ & $\mathrm{PP}$ & -0.418 & 0.17 & Wain et al. [19] \\
\hline PKHD1 & rs13205180 & 6 & $51,967,696$ & $\mathrm{~T}$ & DBP & 0.168 & 0.34 & $\begin{array}{l}\text { Hoffmann et al. [8], } \\
\text { Warren et al. [26] }\end{array}$ \\
\hline PKN2-AS1 & rs61767086 & 1 & $88,600,899$ & G & $\mathrm{PP}$ & 0.413 & 0.14 & Wain et al. [27] \\
\hline PLCB1 & rs6108168 & 20 & $8,645,624$ & A & DBP & -0.211 & 0.38 & Warren et al. [26] \\
\hline PLCD3 & rs12946454 & 17 & $45,130,754$ & $\mathrm{~T}$ & SBP & 0.28 & 0.21 & Newton-Cheh et al. [10] \\
\hline PLCE1 & rs932764 & 10 & $94,136,183$ & G & SBP & 0.484 & 0.43 & Johnson et al. [30] \\
\hline PLCE1 & rs932764 & 10 & $94,136,183$ & G & SBP & 0.484 & 0.44 & Ehret et al. [18] \\
\hline PLEKHA7 & rs 177542 & 11 & $16,901,107$ & A & DBP & 0.243 & 0.50 & Wain et al. [27] \\
\hline PLEKHA7-NUCB2 & rs381815 & 11 & $16,880,721$ & $\mathrm{~T}$ & SBP & 0.84 & 0.21 & Levy et al. [9] \\
\hline PLEKHG1 & rs17080102 & 6 & $150,683,634$ & $\mathrm{C}$ & DBP & -0.74 & 0.12 & Franceschini et al. [24] \\
\hline PNPT1 & rs1975487 & 2 & $55,581,918$ & $\mathrm{~A}$ & DBP & -0.217 & 0.32 & Ehret et al. [12] \\
\hline POC5-SV2C & rs10078021 & 5 & $75,742,606$ & $\mathrm{~T}$ & DBP & -0.164 & 0.46 & $\begin{array}{l}\text { Hoffmann et al. [8], } \\
\text { Warren et al. [26] }\end{array}$ \\
\hline$P P L$ & rs12921187 & 16 & $4,893,018$ & $\mathrm{~T}$ & DBP & -0.174 & 0.41 & $\begin{array}{l}\text { Hoffmann et al. [8], } \\
\text { Warren et al. [26] }\end{array}$ \\
\hline$P P P 2 R 5 E$ & rs8016306 & 14 & $63,461,828$ & A & SBP & 0.335 & 0.41 & Warren et al. [26] \\
\hline PRDM11 & rs11442819 & 11 & $45,186,590$ & I & $\mathrm{PP}$ & -0.279 & 0.13 & $\begin{array}{l}\text { Hoffmann et al. [8], } \\
\text { Warren et al. [26] }\end{array}$ \\
\hline PRDM16 & rs2493292 & 1 & $3,412,095$ & $\mathrm{~T}$ & SBP & 0.42 & 0.13 & Liu et al. [23] \\
\hline PRDM6-SUMO1P5 & rs337100 & 5 & $123,210,816$ & A & PP & 0.277 & 0.40 & Wain et al. [27] \\
\hline PRDM6-CSNK1G3 & rs13359291 & 5 & $123,140,763$ & A & SBP & 0.53 & 0.28 & Kato et al. [20] \\
\hline PRDM8-FGF5 & rs1902859 & 4 & $80,236,549$ & $\mathrm{C}$ & SBP & 1.34 & 0.41 & Lu et al. [51] \\
\hline PRDM8-FGF5 & rs1458038 & 4 & $80,243,569$ & $\mathrm{~T}$ & DBP & 0.403 & 0.30 & Wain et al. [27] \\
\hline PREX1 & rs6095241 & 20 & $48,692,260$ & A & DBP & -0.168 & 0.46 & Surendran et al. [29] \\
\hline PRKAG1 & rs1126930 & 12 & $49,005,349$ & $\mathrm{C}$ & $\mathrm{PP}$ & 0.5 & 0.02 & Surendran et al. [29] \\
\hline PRKCE & rs11690961 & 2 & $46,136,197$ & A & $\mathrm{PP}$ & 0.34 & 0.04 & $\begin{array}{l}\text { Hoffmann et al. [8], } \\
\text { Warren et al. [26] }\end{array}$ \\
\hline PRKD3 & rs13420463 & 2 & $37,290,423$ & $\mathrm{~A}$ & SBP & 0.356 & 0.49 & $\begin{array}{l}\text { Hoffmann et al. [8], } \\
\text { Warren et al. [26] }\end{array}$ \\
\hline PROCR & rs867186 & 20 & $35,176,751$ & A & DBP & 0.265 & 0.11 & Surendran et al. [29] \\
\hline
\end{tabular}


TABle 1: Continued.

\begin{tabular}{|c|c|c|c|c|c|c|c|c|}
\hline Locus name & SNP & $\begin{array}{l}\text { Chr } \\
\text { ID }\end{array}$ & $\begin{array}{c}\mathrm{Chr} \\
\text { position }\end{array}$ & $\begin{array}{l}\text { Coded } \\
\text { allele }\end{array}$ & $\begin{array}{l}\text { Best } \\
\text { trait }\end{array}$ & $\begin{array}{l}\text { Effect size of best } \\
\text { trait (OR beta) }\end{array}$ & $\begin{array}{l}\text { Coded allele } \\
\text { frequency }\end{array}$ & Reporting article \\
\hline PRRC2A-BAG6 & rs151168737 & 6 & $31,638,615$ & $\mathrm{~A}$ & DBP & 0.249 & 0.46 & Wain et al. [27] \\
\hline PSMD5 & rs10760117 & 9 & $120,824,459$ & $\mathrm{~T}$ & SBP & 0.334 & 0.42 & $\begin{array}{c}\text { Ehret et al. [12], } \\
\text { Liu et al. [23] }\end{array}$ \\
\hline$P Y Y$ & rs62080325 & 17 & $43,983,263$ & $\mathrm{~A}$ & $\mathrm{PP}$ & -0.186 & 0.21 & Warren et al. [26] \\
\hline$R A B G A P 1$ & rs 10818775 & 9 & $122,993,292$ & $\mathrm{C}$ & PP & 0.254 & 0.30 & Hoffmann et al. [8] \\
\hline $\begin{array}{l}\text { RAPSN, PSMC3, } \\
\text { SLC39A13 }\end{array}$ & rs7103648 & 11 & $47,440,232$ & A & DBP & -0.203 & 0.33 & Ehret et al. [12] \\
\hline$R B M 47$ & rs35529250 & 4 & $40,426,074$ & $\mathrm{~T}$ & SBP & -1.537 & $<0.01$ & Surendran et al. [29] \\
\hline RCOR2 & rs 4980532 & 11 & $63,913,247$ & $\mathrm{~T}$ & $\mathrm{PP}$ & 0.301 & 0.56 & Wain et al. [27] \\
\hline RGL3 & rs167479 & 19 & $11,416,089$ & $\mathrm{~T}$ & DBP & -0.33 & 0.49 & $\begin{array}{l}\text { Liu et al. [23], } \\
\text { Surendran et al. [29] }\end{array}$ \\
\hline RNF207 & rs709209 & 1 & $6,218,354$ & A & $\mathrm{PP}$ & 0.199 & 0.36 & Surendran et al. [29] \\
\hline$R P 11-273 G 15.2$ & rs62524579 & 8 & $142,979,538$ & A & DBP & -0.175 & 0.48 & $\begin{array}{l}\text { Hoffmann et al. [8], } \\
\text { Warren et al. [26] }\end{array}$ \\
\hline$R P 11-321 F 6.1$ & rs7178615 & 15 & $66,576,734$ & A & DBP & -0.179 & 0.36 & Warren et al. [26] \\
\hline RP11-435J9.2-TLN2 & rs956006 & 15 & $62,516,340$ & $\mathrm{C}$ & $\mathrm{PP}$ & 0.188 & 0.23 & Hoffmann et al. [8] \\
\hline$R P 11-439 C 8.2$ & rs143112823 & 3 & $154,990,178$ & A & DBP & -0.403 & 0.06 & $\begin{array}{l}\text { Hoffmann et al. [8], } \\
\text { Warren et al. [26] }\end{array}$ \\
\hline RP11-61O1.1 & rs9323988 & 14 & $98,121,293$ & $\mathrm{~T}$ & $\mathrm{PP}$ & -0.212 & 0.29 & $\begin{array}{l}\text { Hoffmann et al. [8], } \\
\text { Warren et al. [26] }\end{array}$ \\
\hline $\begin{array}{l}R P 4-710 M 16.1-P P A P 2 B- \\
P L P P 3\end{array}$ & rs112557609 & 1 & $56,111,252$ & A & $\mathrm{PP}$ & 0.227 & 0.22 & $\begin{array}{l}\text { Hoffmann et al. [8], } \\
\text { Warren et al. [26] }\end{array}$ \\
\hline RPL34P18-CDH17 & rs7006531 & 8 & $94,098,516$ & G & $\mathrm{BP}$ & NA & NR & Liang et al. [35] \\
\hline RPL35P4-LOC107986733 & rs10279895 & 7 & $27,288,591$ & G & DBP & 0.7553 & NR & Liang et al. [35] \\
\hline RPL35P4-LOC107986733 & rs 11563582 & 7 & $27,312,031$ & A & $\mathrm{BP}$ & NA & NR & Liang et al. [35] \\
\hline RPL6-PTPN11-ALDH2 & rs11066280 & 12 & $112,379,979$ & $\mathrm{~T}$ & DBP & 1.01 & 0.04 & Kato et al. [22] \\
\hline RPS29P9-LOC102724714 & rs3845811 & 2 & $207,656,788$ & G & SBP & 0.284 & 0.43 & Wain et al. [27] \\
\hline RRAS & rs61760904 & 19 & $49,636,675$ & $\mathrm{~T}$ & SBP & 1.499 & $<0.01$ & Surendran et al. [29] \\
\hline RSPO3 & rs13209747 & 6 & $126,794,309$ & $\mathrm{~T}$ & DBP & 0.56 & 0.35 & Franceschini et al. [24] \\
\hline$R Y K$ & rs9859176 & 3 & $134,281,183$ & $\mathrm{~T}$ & SBP & 0.322 & 0.25 & $\begin{array}{l}\text { Hoffmann et al. [8], } \\
\text { Warren et al. [26] }\end{array}$ \\
\hline SBNO1 & rs1060105 & 12 & $123,321,672$ & $\mathrm{~T}$ & DBP & -0.182 & 0.18 & Surendran et al. [29] \\
\hline SCAI-PPP6C & rs72765298 & 9 & $125,138,717$ & $\mathrm{~T}$ & $\mathrm{PP}$ & -0.374 & 0.06 & $\begin{array}{l}\text { Hoffmann et al. [8], } \\
\text { Warren et al. [26] }\end{array}$ \\
\hline SDCCAG8 & rs953492 & 1 & $243,307,890$ & A & DBP & 0.22 & 0.49 & $\begin{array}{l}\text { Hoffmann et al. [8], } \\
\text { Warren et al. [26] }\end{array}$ \\
\hline SENP2 & rs12374077 & 3 & $185,599,886$ & $\mathrm{C}$ & DBP & 0.163 & 0.42 & $\begin{array}{l}\text { Hoffmann et al. [8], } \\
\text { Warren et al. [26] }\end{array}$ \\
\hline SEPT9 & rs57927100 & 17 & $77,321,218$ & G & SBP & -0.489 & 0.01 & Wain et al. [27] \\
\hline SETBP1 & rs12958173 & 18 & $44,562,012$ & A & SBP & 0.386 & 0.25 & Ehret et al. [12] \\
\hline $\mathrm{SH} 2 \mathrm{~B} 3$ & rs3184504 & 12 & $111,446,804$ & $\mathrm{~T}$ & SBP & 0.75 & 0.33 & $\begin{array}{l}\text { Levy et al. [9], } \\
\text { Newton-Cheh et al. [10] }\end{array}$ \\
\hline SLC12A9 & rs7801190 & 7 & $100,860,471$ & $\mathrm{C}$ & $\mathrm{BP}$ & 1.31 & 0.72 & Lettre et al. [39] \\
\hline SLC14A2 & rs7236548 & 18 & $45,517,785$ & A & $\mathrm{PP}$ & 0.352 & 0.3 & $\begin{array}{l}\text { Hoffmann et al. [8], } \\
\text { Warren et al. [26] }\end{array}$ \\
\hline SLC20A2 & rs2978456 & 8 & $42,467,247$ & $\mathrm{~T}$ & $\mathrm{PP}$ & -0.188 & 0.45 & $\begin{array}{l}\text { Hoffmann et al. [8], } \\
\text { Warren et al. [26] }\end{array}$ \\
\hline$S L C 24 A 3$ & rs6081613 & 20 & $19,485,263$ & A & $\mathrm{PP}$ & 0.263 & 0.31 & $\begin{array}{l}\text { Hoffmann et al. [8], } \\
\text { Warren et al. [26] }\end{array}$ \\
\hline SLC35F1 & rs9372498 & 6 & $118,251,323$ & A & DBP & 0.334 & 0.07 & $\begin{array}{l}\text { Hoffmann et al. [8], } \\
\text { Warren et al. [26] }\end{array}$ \\
\hline
\end{tabular}


TABle 1: Continued.

\begin{tabular}{|c|c|c|c|c|c|c|c|c|}
\hline Locus name & SNP & $\begin{array}{l}\text { Chr } \\
\text { ID }\end{array}$ & $\begin{array}{c}\text { Chr } \\
\text { position }\end{array}$ & $\begin{array}{c}\text { Coded } \\
\text { allele }\end{array}$ & $\begin{array}{l}\text { Best } \\
\text { trait }\end{array}$ & $\begin{array}{l}\text { Effect size of best } \\
\text { trait (OR beta) }\end{array}$ & $\begin{array}{c}\text { Coded allele } \\
\text { frequency }\end{array}$ & Reporting article \\
\hline SLC39A8 & rs13107325 & 4 & $102,267,552$ & $\mathrm{~T}$ & DBP & -0.684 & 0 & Johnson et al. [30] \\
\hline SLC4A7 & rs11716531 & 3 & $27,415,717$ & A & DBP & 0.213 & 0.237 & Wain et al. [27] \\
\hline SLC4A7 & rs13082711 & 3 & $27,496,418$ & $\mathrm{~T}$ & DBP & -0.238 & 0.12 & Johnson et al. [30] \\
\hline SLC $8 A 1$ & rs4952611 & 2 & $40,340,603$ & $\mathrm{~T}$ & DBP & -0.157 & 0.34 & Warren et al. [26] \\
\hline SMARCA2-VLDLR & rs872256 & 9 & $2,496,480$ & $\mathrm{~T}$ & SBP & 0.096 & 0.43 & Parmar et al. [37] \\
\hline SNORD32B & rs926552 & 6 & $29,580,312$ & $\mathrm{~T}$ & DBP & -0.31 & 0.07 & Liu et al. [23] \\
\hline SNX31 & rs2978098 & 8 & $100,664,447$ & $\mathrm{~A}$ & DBP & 0.165 & 0.34 & Warren et al. [26] \\
\hline SOX6 & rs4757391 & 11 & $16,281,393$ & $\mathrm{C}$ & DBP & 0.49 & 0.28 & Lu et al. [51] \\
\hline SSPN & rs6487543 & 12 & $26,285,256$ & $\mathrm{~A}$ & SBP & 0.3 & 0.46 & Warren et al. [26] \\
\hline ST7L-CAPZA1-MOV10 & rs2932538 & 1 & $112,673,921$ & G & DBP & 0.24 & 0.17 & Johnson et al. [30] \\
\hline STK39 & rs6749447 & 2 & $168,184,876$ & G & SBP & 3 & 0.48 & Wang et al. [40] \\
\hline SUGCT & rs76206723 & 7 & $40,408,372$ & A & PP & -0.346 & 0.18 & $\begin{array}{l}\text { Hoffmann et al. [8], } \\
\text { Warren et al. [26] }\end{array}$ \\
\hline SULT1C3 & rs6722745 & 2 & $108,258,788$ & $\mathrm{C}$ & SBP & 0.28 & 0.4 & Liu et al. [23] \\
\hline SVEP1 & rs 111245230 & 9 & $110,407,495$ & $\mathrm{C}$ & SBP & 0.94 & 0.03 & Liu et al. [23] \\
\hline SWAP70 & rs2649044 & 11 & $9,742,422$ & $\mathrm{~T}$ & DBP & 0.2 & 0.547 & Wain et al. [27] \\
\hline TBC1D1-FLJ13197 & rs2291435 & 4 & $38,385,774$ & $\mathrm{~T}$ & SBP & -0.378 & 0.4 & Ehret et al. [12] \\
\hline TBX5-TBX3 & rs 2384550 & 12 & $114,914,926$ & A & DBP & -0.35 & 0.29 & $\begin{array}{l}\text { Levy et al. [9], } \\
\text { Kato et al. [22] }\end{array}$ \\
\hline TCF7L1 & rs11689667 & 2 & $85,264,242$ & $\mathrm{~T}$ & $\mathrm{PP}$ & 0.176 & 0.28 & $\begin{array}{l}\text { Hoffmann et al. [8], } \\
\text { Warren et al. [26] }\end{array}$ \\
\hline TCF7L2 & rs34872471 & 10 & $112,994,312$ & $\mathrm{~T}$ & $\mathrm{PP}$ & -0.226 & 0.24 & Hoffmann et al. [8] \\
\hline TEX41 & rs1438896 & 2 & $144,888,505$ & $\mathrm{~T}$ & DBP & 0.234 & 0.3 & $\begin{array}{l}\text { Hoffmann et al. [8], } \\
\text { Warren et al. [26] }\end{array}$ \\
\hline TEX41 & rs55944332 & 2 & $144,969,054$ & G & DBP & 0.267 & 0.24 & Wain et al. [27] \\
\hline TFAP2D & rs78648104 & 6 & $50,715,296$ & $\mathrm{~T}$ & SBP & -0.481 & 0.09 & Warren et al. [26] \\
\hline TM6SF1 & rs2034618 & 15 & $83,130,880$ & $\mathrm{C}$ & DBP & 0.21 & 0.22 & Hoffmann et al. [8] \\
\hline TMEM161B & rs10059921 & 5 & $88,218,698$ & $\mathrm{~T}$ & SBP & -0.526 & 0.06 & $\begin{array}{l}\text { Hoffmann et al. [8], } \\
\text { Warren et al. [26] }\end{array}$ \\
\hline $\begin{array}{l}\text { TMEM194B-NEMP2- } \\
\text { NAB1 }\end{array}$ & rs7592578 & 2 & $190,574,865$ & $\mathrm{~T}$ & DBP & -0.240 & 0.18 & $\begin{array}{l}\text { Hoffmann et al. [8], } \\
\text { Warren et al. [26] }\end{array}$ \\
\hline TNRC6A & rs11639856 & 16 & $24,777,324$ & A & SBP & -0.37 & 0.17 & Liu et al. [23] \\
\hline TNRC6B & rs470113 & 22 & $40,333,610$ & A & $\mathrm{PP}$ & -0.253 & 0.21 & Surendran et al. [29] \\
\hline TNS1 & rs1063281 & 2 & $217,804,009$ & $\mathrm{~T}$ & DBP & -0.200 & 0.43 & $\begin{array}{l}\text { Hoffmann et al. [8], } \\
\text { Warren et al. [26] }\end{array}$ \\
\hline$T N X B$ & rs2021783 & 6 & $32,077,074$ & $\mathrm{C}$ & DBP & 0.49 & 0.79 & Lu et al. [51] \\
\hline$T N X B$ & rs185819 & 6 & $32,082,290$ & $\mathrm{C}$ & SBP & 0.365 & 0.513 & Wain et al. [27] \\
\hline TP53-SLC2A4 & rs78378222 & 17 & $7,668,434$ & $\mathrm{~T}$ & $\mathrm{PP}$ & 0.904 & 0 & $\begin{array}{l}\text { Hoffmann et al. [8], } \\
\text { Warren et al. [26] }\end{array}$ \\
\hline TRAPPC9 & rs4288356 & 8 & $140,045,627$ & $\mathrm{~A}$ & $\mathrm{PP}$ & 0.224 & 0.615 & Wain et al. [27] \\
\hline TRAPPC9 & rs 4454254 & 8 & $140,049,929$ & $\mathrm{~A}$ & PP & -0.261 & 0.45 & Warren et al. [26] \\
\hline TRIM36 & rs10077885 & 5 & $115,054,424$ & $\mathrm{~A}$ & DBP & -0.194 & 0.42 & Ehret et al. [12] \\
\hline UBA52P4-LOC105377005 & rs820430 & 3 & $27,507,409$ & $\mathrm{~A}$ & SBP & 0.76 & 0.32 & Lu et al. [51] \\
\hline ULK4 & rs7651190 & 3 & $41,724,463$ & G & $\mathrm{BP}$ & NA & NR & Liang et al. [35] \\
\hline ULK4 & rs9815354 & 3 & $41,912,651$ & $\mathrm{~A}$ & DBP & 0.6 & 0.17 & Levy et al. [9] \\
\hline ULK4 & rs7372217 & 3 & $41,948,630$ & G & $\mathrm{BP}$ & NA & NR & Liang et al. [35] \\
\hline$U M O D$ & rs13333226 & 16 & $20,354,332$ & NA & HTN & NA & 0.24 & Padmanabhan et al. $[25$ \\
\hline VAC14 & rs117006983 & 16 & $70,721,707$ & A & PP & 0.986 & 0 & Warren et al. [26] \\
\hline WNT3A & rs2760061 & 1 & $228,003,374$ & A & DBP & 0.23 & 0.35 & $\begin{array}{l}\text { Hoffmann et al. [8], } \\
\text { Warren et al. [26] }\end{array}$ \\
\hline
\end{tabular}


TABLe 1: Continued.

\begin{tabular}{|c|c|c|c|c|c|c|c|c|}
\hline Locus name & SNP & $\begin{array}{l}\mathrm{Chr} \\
\text { ID }\end{array}$ & $\begin{array}{c}\mathrm{Chr} \\
\text { position }\end{array}$ & $\begin{array}{l}\text { Coded } \\
\text { allele }\end{array}$ & $\begin{array}{l}\text { Best } \\
\text { trait }\end{array}$ & $\begin{array}{l}\text { Effect size of best } \\
\text { trait (OR beta) }\end{array}$ & $\begin{array}{l}\text { Coded allele } \\
\text { frequency }\end{array}$ & Reporting article \\
\hline XKR6 & rs10107145 & 8 & $10,900,703$ & $\mathrm{G}$ & SBP & 0.361 & 0.528 & Wain et al. [27] \\
\hline XRCC6 & rs73161324 & 22 & $41,642,782$ & $\mathrm{~T}$ & $\mathrm{PP}$ & 0.496 & 0.02 & Warren et al. [26] \\
\hline ZBTB38 & rs16851397 & 3 & $141,415,976$ & A & $\mathrm{DBP}$ & -0.493 & 0.05 & Surendran et al. [29] \\
\hline $\mathrm{ZC} 3 \mathrm{HC} 1$ & rs11556924 & 7 & $130,023,656$ & $\mathrm{~T}$ & $\mathrm{DBP}$ & -0.214 & 0.27 & Ehret et al. [12] \\
\hline$Z F A T$ & rs894344 & 8 & $134,600,502$ & A & SBP & -0.258 & 0.47 & Warren et al. [26] \\
\hline ZNF101 & rs2304130 & 19 & $19,678,719$ & A & $\mathrm{DBP}$ & -0.292 & 0.11 & Surendran et al. [29] \\
\hline ZNF318-ABCC10 & rs10948071 & 6 & $43,312,975$ & $\mathrm{~T}$ & $\mathrm{PP}$ & -0.38 & 0.43 & Ganesh et al. [34] \\
\hline ZNF385B & rs13407401 & 2 & $179,850,979$ & A & SBP & 0.434 & 0.291 & Wain et al. [27] \\
\hline ZNF638 & rs3771371 & 2 & $71,400,409$ & $\mathrm{~T}$ & $\mathrm{PP}$ & -0.160 & 0.37 & $\begin{array}{l}\text { Hoffmann et al. [8], } \\
\text { Warren et al. [26] }\end{array}$ \\
\hline ZNF652 & rs12940887 & 17 & $49,325,445$ & $\mathrm{~T}$ & DBP & 0.26 & 0.374 & Wain et al. [27] \\
\hline ZNF652 & rs16948048 & 17 & $49,363,104$ & G & DBP & 0.39 & 0.29 & Newton-Cheh et al. [10] \\
\hline ZNRF3 & rs4823006 & 22 & $29,055,683$ & G & SBP & -0.33 & 0.45 & Liu et al. [23] \\
\hline
\end{tabular}

SBP: systolic blood pressure; DBP: diastolic blood pressure; PP: pulse pressure; MAP: mean arterial pressure; HTN: hypertension; NR: not recorded; NA: not available.

3.3. Corticosteroidogenesis. Surprisingly in the sense that corticosteroids can highly affect blood pressure, only 2 cytochrome P450 enzyme genes involved with corticosteroidogenesis have been linked to hypertension by GWASs-CYP17A1 and CYP21A2. And of that, only SNPs in the CYP17A1 gene have been replicated, though even then with inconsistent results. CYP17A1 encodes $17 \alpha$-hydroxylase which is essential to the synthesis of cortisol precursors. Therefore, alteration of this gene can cause a deficiency in $17 \alpha$-hydroxylase and thus cortisol, which affects blood pressure [57]. Supporting the role of CYP17A1 in blood pressure regulation is the SNP rs11191548, a SNP near the CYP17A1 gene that has been consistently associated with blood pressure in both East Asian cohorts and Caucasian cohorts [10, 17, 18, 58-60]. Patients harboring the risky $\mathrm{C}$ allele had lower PRA and $\mathrm{K}+$ levels similar to patients with $17 \alpha$-hydroxylase deficiency, suggesting that the SNP (which is actually in the noncoding region of the gene $C N N M 2$ ) has an effect on the enzymatic activity of CYP17A1 [58]. One hypothesis as to why inconsistent results occur with GWAS is if the association found between the lead SNP is indirect whereby the signal produced is actually caused by a synthetically linked rarer variant in linkage disequilibrium with the identified tag SNP. This could be the case with the lead SNP rs1004467 which was identified from the CHARGE + Global BPgen metaanalysis [9]. In an Oriental cohort (from Korea), rs1004467 was found to have a modest association with hypertension in prediabetic subjects and a significant association with augmentation index in diabetic subjects [61]. However, in another Oriental cohort with similar ethnic background (from China), rs1004467 association with hypertension/ blood pressure was not found in children [62]. As such, perhaps the causal SNP is not rs1004467 as identified by the initial GWAS meta-analysis but a tag SNP with poor penetrance. Interestingly, rs1004467 is in linkage disequilibrium with rs138009835, a functional SNP located 1800 bases upstream of the transcription site of CYP17A1. In vitro gene reporter gene assays and clinical functional experiments found the minor alleles to have reduced mRNA expression of CYP17A1 and reduced aldosterone excretion [63]. To note, both rs1004467 and rs11191548 are associated with a reduction in both visceral and subcutaneous fat mass in Japanese women [64].

3.4. Vascular Tone. Interestingly, although only one of the fifteen monogenic hypertension genes is postulated to mediate an effect through the vasculature, SNPs associated with blood pressure and primary hypertension are enriched in genes that are expressing their proteins in vascular smooth muscle and endothelial cells $[11,12,65-67]$. This is consistent with vascular tone playing a primary role in blood pressure regulation. Many of these genes, however, may have been reported as the causal genes due to their proximity to the SNP in question and their likelihood of playing a role in blood pressure regulation rather than due to real functional data [68]. For example, the reported gene for rs7129220, a SNP downstream to the $A D M$ gene in the noncoding RNA CAND1.11 gene, was the $A D M$ gene as adrenomedullin the protein encoded by $A D M$ plays a role in vasodilation [69]. Oppositely, the reported genes for rs633185 are FLJ32810-TMEM133, even though the SNP is within the intron of ARHGAP42 (Table 1). As a candidate gene for blood pressure regulation, ARHGAP42 has many functional evidence to be the causal gene as reduced expression of ARHGAP42 in mice elevated blood pressure [70]. To note, rs633185 is in high linkage disequilibrium with rs604723, another SNP in the intron of ARHGAP42, and the minor $\mathrm{T}$ allele is a functional variant that increases ARHGAP42 expression by promoting serum response factor binding to a smooth muscle-selective regulatory element [71]. Based on this strong functional data, rs604723 is most likely the causative SNP at this locus. rs6271 in exon 11 of the $D B H$ gene on the other hand is one of the rare times where GWASs had managed to directly identify a missense variant which is probably damaging to the protein dopamine $\beta$-hydroxylase according to PolyPhen-2 prediction [72]. Concurringly, severe orthostatic 
syndrome (postural hypotension) were found to be caused by truncating, splice site, or missense mutations in the $D B H$ gene [73].

\section{Conclusion}

Although some of the SNPs identified by GWAS on primary hypertension associates with similar biological pathways as Mendelian or early-onset forms of hypertension (validating the study approach), none of the SNPs identified had a large size effect $(\leq 1 \mathrm{mmHg})$ to be of significance to an individual patient. The ultimate goals of performing these GWASs are to determine the genetic factors regulating blood pressure that can be used to make predictions about who is at risk of developing hypertension and to identify the biological pathways of the disease allowing for identification of novel targets for treatment or even prevention strategies. As currently no direct clinical application of these GWAS findings can be made, it is still debatable whether GWAS is the best approach to identify the biological underpinnings of primary hypertension. Even though yet-to-be-discovered Oriental-specific loci or rare SNPs that might have larger effect size may increase the variance for blood pressure that can be explained by genetic variation, information on epigenetic modulation (e.g., DNA methylation, posttranslational modifications of proteins, or even gut microbiota $[20,74-78]$ ) may still be needed to explain the total heritability of raised blood pressure which cannot be captured by GWASs.

\section{Conflicts of Interest}

The authors declare that there is no conflict of interest regarding the publication of this paper.

\section{Acknowledgments}

The authors thank Ms. Syahirah Kaja Mohideen for her help in preparing this manuscript. Elena Aisha Binti Azizan's research was supported by the Malaysian Minister of Higher Education grant (FRGS/1/2015/SKK08/UKM/02/3). Afifah Binti Azam's salary is supported by the National University of Malaysia (UKM) grant (GUP-2016-083) awarded to Elena Aisha Binti Azizan.

\section{References}

[1] P. Kearney, M. Whelton, K. Reynolds, P. Muntner, P. Whelton, and J. He, "Global burden of hypertension: analysis of worldwide data," The Lancet, vol. 365, no. 9455, pp. 217-223, 2005.

[2] Global Health Observatory (GHO) data, "Raised blood pressure: situation and trends," http://www.who.int/gho/ncd/risk_ factors/blood_pressure_prevalence_text/en.

[3] Clinical Practice Guidelines, Management of Hypertension, Ministry of Health, Malaysia, 4th edition, 2013.

[4] O. A. Carretero and S. Oparil, "Essential hypertension: part I: definition and etiology," Circulation, vol. 101, no. 3, pp. 329$335,2000$.

[5] J. B. Meigs, A. K. Manning, C. S. Fox et al., "Genome-wide association with diabetes-related traits in the Framingham
Heart Study," BMC Medical Genetics, vol. 8, article S16, Supplement 1, 2007.

[6] M. O. M. Tanira and K. A. Al Balushi, "Genetic variations related to hypertension: a review," Journal of Human Hypertension, vol. 19, no. 1, pp. 7-19, 2005.

[7] R. P. Lifton, "Molecular genetics of human blood pressure variation," Science, vol. 272, no. 5262, pp. 676-680, 1996.

[8] T. J. Hoffmann, G. B. Ehret, P. Nandakumar et al., "Genomewide association analyses using electronic health records identify new loci influencing blood pressure variation," Nature Genetics, vol. 49, no. 1, pp. 54-64, 2017.

[9] D. Levy, G. B. Ehret, K. Rice et al., "Genome-wide association study of blood pressure and hypertension," Nature Genetics, vol. 41, no. 6, pp. 677-687, 2009.

[10] C. Newton-Cheh, T. Johnson, V. Gateva et al., "Eight blood pressure loci identified by genome-wide association study of 34,433 people of European ancestry," Nature Genetics, vol. 41, no. 6, pp. 666-676, 2009.

[11] G. B. Ehret, "Genetics of hypertension," in Hypertension: A Companion to Braunwald's Heart Disease E-Book, p. pp. 5259, Elsevier, USA, 2017.

[12] G. B. Ehret, T. Ferreira, D. I. Chasman et al., "The genetics of blood pressure regulation and its target organs from association studies in 342,415 individuals," Nature Genetics, vol. 48, no. 10, pp. 1171-1184, 2016.

[13] G. L. Hong, X. Z. Chen, Y. Liu et al., "Genetic variations in MOV10 and CACNB2 are associated with hypertension in a Chinese Han population," Genetics and Molecular Research, vol. 12, no. 4, pp. 6220-6227, 2013.

[14] A. R. Hong, J. H. Kim, Y. S. Song et al., "Genetics of aldosterone-producing adenoma in Korean patients," PLoS One, vol. 11, no. 1, article e0147590, 2016.

[15] F. Takeuchi, M. Isono, T. Katsuya et al., "Blood pressure and hypertension are associated with 7 loci in the Japanese population," Hypertension, vol. 121, no. 21, pp. 2302-2309, 2010.

[16] C. Liu, H. Li, Q. Qi et al., "Common variants in or near FGF5, CYP17A1 and MTHFR genes are associated with blood pressure and hypertension in Chinese Hans," Journal of Hypertension, vol. 29, no. 1, pp. 70-75, 2011.

[17] B. Xi, Y. Shen, K. H. Reilly, X. Wang, and J. Mi, "Recapitulation of four hypertension susceptibility genes (CSK, CYP17A1, MTHFR, and FGF5) in East Asians," Metabolism, vol. 62, no. 2, pp. 196-203, 2013.

[18] International Consortium for Blood Pressure Genome-Wide Association Studies, G. B. Ehret, P. B. Munroe et al., "Genetic variants in novel pathways influence blood pressure and cardiovascular disease risk," Nature, vol. 478, no. 7367, pp. 103-109, 2011.

[19] L. V. Wain, G. C. Verwoert, P. F. O'Reilly et al., "Genome-wide association study identifies six new loci influencing pulse pressure and mean arterial pressure," Nature Genetics, vol. 43, no. 10, pp. 1005-1011, 2011.

[20] N. Kato, M. Loh, F. Takeuchi et al., "Trans-ancestry genomewide association study identifies 12 genetic loci influencing blood pressure and implicates a role for DNA methylation," Nature Genetics, vol. 47, no. 11, pp. 1282-1293, 2015.

[21] X. Lu, L. Wang, X. Lin et al., "Genome-wide association study in Chinese identifies novel loci for blood pressure and hypertension," Human Molecular Genetics, vol. 24, no. 3, pp. 865874, 2015. 
[22] N. Kato, F. Takeuchi, Y. Tabara et al., "Meta-analysis of genome-wide association studies identifies common variants associated with blood pressure variation in east Asians," Nature Genetics, vol. 43, no. 6, pp. 531-538, 2011.

[23] X. Liu, C. Hu, M. Bao et al., "Genome wide association study identifies L3MBTL4 as a novel susceptibility gene for hypertension," Scientific Reports, vol. 6, no. 1, article 30811, 2016.

[24] N. Franceschini, E. Fox, Z. Zhang et al., "Genome-wide association analysis of blood-pressure traits in African-ancestry individuals reveals common associated genes in African and non-African populations," The American Journal of Human Genetics, vol. 93, no. 3, pp. 545-554, 2013.

[25] S. Padmanabhan, O. Melander, T. Johnson et al., "Genomewide association study of blood pressure extremes identifies variant near UMOD associated with hypertension," PLOS Genetics, vol. 6, no. 10, article e1001177, 2010.

[26] H. R. Warren, E. Evangelou, C. P. Cabrera et al., "Genomewide association analysis identifies novel blood pressure loci and offers biological insights into cardiovascular risk," Nature Genetics, vol. 49, no. 3, pp. 403-415, 2017.

[27] L. V. Wain, A. Vaez, R. Jansen et al., "Novel blood pressure locus and gene discovery using genome-wide association study and expression data sets from blood and the kidney," Hypertension, vol. 70, no. 3, pp. e4-e19, 2017.

[28] S. S. Lim, T. Vos, A. D. Flaxman et al., "A comparative risk assessment of burden of disease and injury attributable to 67 risk factors and risk factor clusters in 21 regions, 1990-2010: a systematic analysis for the Global Burden of Disease Study 2010," The Lancet, vol. 380, no. 9859, pp. 2224-2260, 2012.

[29] P. Surendran, F. Drenos, R. Young et al., "Trans-ancestry meta-analyses identify rare and common variants associated with blood pressure and hypertension," Nature Genetics, vol. 48, no. 10, pp. 1151-1161, 2016.

[30] A. D. Johnson, C. Newton-Cheh, D. I. Chasman et al., "Association of hypertension drug target genes with blood pressure and hypertension in 86588 individuals," Hypertension, vol. 57, no. 5, pp. 903-910, 2011.

[31] T. N. Kelly, F. Takeuchi, Y. Tabara et al., "Genome-wide association study meta-analysis reveals transethnic replication of mean arterial and pulse pressure loci," Hypertension, vol. 62, no. 5, pp. 853-859, 2013.

[32] X. Zhu, T. Feng, B. O. Tayo et al., "Meta-analysis of correlated traits via summary statistics from GWASs with an application in hypertension," The American Journal of Human Genetics, vol. 96, no. 1, pp. 21-36, 2015.

[33] J. E. Ho, L. Daniel, R. Lynda, A. D. Johnson, P. M. Ridker, and D. I. Chasman, "Discovery and replication of novel blood pressure genetic loci in the Women's Genome Health Study," Journal of Hypertension, vol. 29, no. 1, pp. 62-69, 2011.

[34] S. K. Ganesh, D. I. Chasman, M. G. Larson et al., "Effects of long-term averaging of quantitative blood pressure traits on the detection of genetic associations," The American Journal of Human Genetics, vol. 95, no. 1, pp. 49-65, 2014.

[35] J. Liang, T. H. Le, D. R. V. Edwards et al., "Single-trait and multi-trait genome-wide association analyses identify novel loci for blood pressure in African-ancestry populations," PLoS Genetics, vol. 13, no. 5, article e1006728, 2017.

[36] E. R. Fox, J. H. Young, Y. Li et al., “Association of genetic variation with systolic and diastolic blood pressure among African Americans: the Candidate Gene Association Resource study," Human Molecular Genetics, vol. 20, no. 11, pp. 2273 2284, 2011.

[37] P. G. Parmar, H. R. Taal, N. J. Timpson et al., "International Genome-Wide Association Study consortium identifies novel loci associated with blood pressure in children and adolescents," Circulation: Cardiovascular Genetics, vol. 9, no. 3, pp. 266-278, 2016.

[38] J. Simino, G. Shi, J. C. Bis et al., "Gene-age interactions in blood pressure regulation: a large-scale investigation with the CHARGE, Global BPgen, and ICBP Consortia," The American Journal of Human Genetics, vol. 95, no. 1, pp. 2438, 2014.

[39] G. Lettre, C. D. Palmer, T. Young et al., "Genome-wide association study of coronary heart disease and its risk factors in 8,090 African Americans: the NHLBI CARe Project," PLoS Genetics, vol. 7, no. 2, article e1001300, 2011.

[40] Y. Wang, J. R. O'Connell, P. F. McArdle et al., "Whole-genome association study identifies STK39 as a hypertension susceptibility gene," Proceedings of the National Academy of Sciences, vol. 106, no. 1, pp. 226-231, 2009.

[41] G. B. Ehret and M. J. Caulfield, "Genes for blood pressure: an opportunity to understand hypertension," European Heart Journal, vol. 34, no. 13, pp. 951-961, 2013.

[42] E. A. B. Azizan, H. Poulsen, P. Tuluc et al., "Somatic mutations in ATP1A1 and CACNA1D underlie a common subtype of adrenal hypertension," Nature Genetics, vol. 45, no. 9, pp. 1055-1060, 2013.

[43] P. G. Maass, A. Aydin, F. C. Luft et al., "PDE3A mutations cause autosomal dominant hypertension with brachydactyly," Nature Genetics, vol. 47, no. 6, pp. 647-653, 2015.

[44] U. I. Scholl, G. Stölting, C. Nelson-Williams et al., "Recurrent gain of function mutation in calcium channel CACNA1H causes early-onset hypertension with primary aldosteronism," eLife, vol. 4, article e06315, 2015.

[45] M. D. Tobin, M. Tomaszewski, P. S. Braund et al., "Common variants in genes underlying monogenic hypertension and hypotension and blood pressure in the general population," Hypertension, vol. 51, no. 6, pp. 1658-1664, 2008.

[46] Y. X. Gao, C. A. Yu, J. H. Lu et al., "ADAMTS-7 expression increases in the early stage of angiotensin II-induced renal injury in elderly mice," Kidney \& Blood Pressure Research, vol. 38, no. 1, pp. 121-131, 2013.

[47] A. W. Cowley Jr., C. Yang, V. Kumar et al., "Pappa2 is linked to salt-sensitive hypertension in Dahl S rats," Physiological Genomics, vol. 48, no. 1, pp. 62-72, 2016.

[48] D. A. Braun, M. Schueler, J. Halbritter et al., "Whole exome sequencing identifies causative mutations in the majority of consanguineous or familial cases with childhood-onset increased renal echogenicity," Kidney International, vol. 89, no. 2, pp. 468-475, 2016.

[49] G. Xu, X. Lu, T. Huang, and J. Fan, “ARHGAP24 inhibits cell cycle progression, induces apoptosis and suppresses invasion in renal cell carcinoma," Oncotarget, vol. 7, no. 32, pp. 5182951839, 2016.

[50] S. Akilesh, H. Suleiman, H. Yu et al., "Arhgap24 inactivates Rac1 in mouse podocytes, and a mutant form is associated with familial focal segmental glomerulosclerosis," The Journal of Clinical Investigation, vol. 121, no. 10, pp. 41274137, 2011.

[51] L. X, L. Wang, S. Chen et al., "Genome-wide association study in Han Chinese identifies four new susceptibility loci 
for coronary artery disease," Nature Genetics, vol. 44, no. 8 , pp. 890-894, 2014.

[52] Y. Kobayashi, N. Hirawa, Y. Tabara et al., "Mice lacking hypertension candidate gene ATP2B1 in vascular smooth muscle cells show significant blood pressure elevation," Hypertension, vol. 59, no. 4, pp. 854-860, 2012.

[53] Y. B. Shin, J. E. Lim, S. M. Ji et al., "Silencing of Atp2b1 increases blood pressure through vasoconstriction," Journal of Hypertension, vol. 31, no. 8, pp. 1575-1583, 2013.

[54] U. I. Scholl, G. Goh, G. Stölting et al., "Somatic and germline CACNA1D calcium channel mutations in aldosteroneproducing adenomas and primary aldosteronism," Nature Genetics, vol. 45, no. 9, pp. 1050-1054, 2013.

[55] K. Nishimoto, S. A. Tomlins, R. Kuick et al., "Aldosteronestimulating somatic gene mutations are common in normal adrenal glands," Proceedings of the National Academy of Sciences, vol. 112, no. 33, pp. E4591-E4599, 2015.

[56] E. A. B. Azizan, M. Murthy, M. Stowasser et al., "Somatic mutations affecting the selectivity filter of KCNJ5 are frequent in 2 large unselected collections of adrenal aldosteronomas," Hypertension, vol. 59, no. 3, pp. 587-591, 2012.

[57] M. Huber, S. Lezius, R. Reibis et al., "A single nucleotide polymorphism near the CYP17A1 gene is associated with left ventricular mass in hypertensive patients under pharmacotherapy," International Journal of Molecular Sciences, vol. 16, no. 8, pp. 17456-17468, 2015.

[58] X. Li, Y. Ling, D. Lu et al., "Common polymorphism rs11191548 near the CYP17A1 gene is associated with hypertension and systolic blood pressure in the Han Chinese population," American Journal of Hypertension, vol. 26, no. 4, pp. 465-472, 2013.

[59] K. Miyaki, N. C. Htun, Y. Song, S. Ikeda, M. Muramatsu, and T. Shimbo, "The combined impact of 12 common variants on hypertension in Japanese men, considering GWAS results," Journal of Human Hypertension, vol. 26, no. 7, pp. 430-436, 2012.

[60] Y. Lin, X. Lai, B. Chen et al., "Genetic variations in CYP17A1, CACNB2 and PLEKHA7 are associated with blood pressure and/or hypertension in She ethnic minority of China," Atherosclerosis, vol. 219, no. 2, pp. 709-714, 2011.

[61] S. J. Yang, S. T. Lee, W. J. Kim et al., "Genetic variation in CYP17A1 is associated with arterial stiffness in diabetic subjects," Experimental Diabetes Research, vol. 2012, Article ID 827172, pp. 1-8, 2012.

[62] B. Xi, Y. Shen, X. Zhao et al., "Association of common variants in/near six genes (ATP2B1, CSK, MTHFR, CYP17A1, STK39 and FGF5) with blood pressure/hypertension risk in Chinese children," Journal of Human Hypertension, vol. 28, no. 1, pp. 32-36, 2014.

[63] L. A. Diver, S. M. MacKenzie, R. Fraser et al., "Common polymorphisms at the CYP17A1 locus associate with steroid phenotype," Hypertension, vol. 67, no. 4, pp. 724-732, 2016.

[64] K. Hotta, A. Kitamoto, T. Kitamoto et al., "Genetic variations in the CYP17A1 and NT5C2 genes are associated with a reduction in visceral and subcutaneous fat areas in Japanese women," Journal of Human Genetics, vol. 57, no. 1, pp. 46-51, 2012.

[65] D. L. Mattson and M. Liang, "Hypertension: from GWAS to functional genomics-based precision medicine," Nature Reviews Nephrology, vol. 13, no. 4, pp. 195-196, 2017.
[66] M. Sano, N. Kuroi, T. Nakayama et al., "Association study of calcitonin-receptor-like receptor gene in essential hypertension," American Journal of Hypertension, vol. 18, no. 3, pp. 403-408, 2005.

[67] C. Lofqvist, J. Chen, K. M. Connor et al., "IGFBP3 suppresses retinopathy through suppression of oxygen-induced vessel loss and promotion of vascular regrowth," Proceedings of the National Academy of Sciences, vol. 104, no. 25, pp. 1058910594, 2007.

[68] G. B. Ehret, "Genome-wide association studies: contribution of genomics to understanding blood pressure and essential hypertension," Current Hypertension Reports, vol. 12, no. 1, pp. 17-25, 2010.

[69] T. Shindo, T. Sakurai, A. Kamiyoshi et al., "Regulation of adrenomedullin and its family peptide by RAMP system - lessons from genetically engineered mice," Current Protein \& Peptide Science, vol. 14, no. 5, pp. 347-357, 2013.

[70] X. Bai, K. C. Lenhart, K. E. Bird et al., “The smooth muscleselective RhoGAP GRAF3 is a critical regulator of vascular tone and hypertension," Nature Communications, vol. 4, p. 2910, 2013.

[71] X. Bai, K. D. Mangum, R. A. Dee et al., "Blood pressure-associated polymorphism controls ARHGAP42 expression via serum response factor DNA binding," The Journal of Clinical Investigation, vol. 127, no. 2, pp. 670-680, 2017.

[72] I. A. Adzhubei, S. Schmidt, L. Peshkin et al., "A method and server for predicting damaging missense mutations," Nature Methods, vol. 7, no. 4, pp. 248-249, 2010.

[73] J. Deinum, G. C. Steenbergen-Spanjers, M. Jansen et al., “ $D B H$ gene variants that cause low plasma dopamine $\beta$ hydroxylase with or without a severe orthostatic syndrome," Journal of Medical Genetics, vol. 41, no. 4, article e38, 2004.

[74] V. V. Lima, F. R. C. Giachini, H. Choi et al., "Impaired vasodilator activity in deoxycorticosterone acetate-salt hypertension is associated with increased protein O-GlcNAcylation," Hypertension, vol. 53, no. 2, pp. 166-174, 2009.

[75] V. V. Lima, C. S. Rigsby, D. M. Hardy, R. C. Webb, and R. C. Tostes, "O-GlcNAcylation: a novel post-translational mechanism to alter vascular cellular signaling in health and disease: focus on hypertension," Journal of the American Society of Hypertension, vol. 3, no. 6, pp. 374-387, 2009.

[76] F. Z. Marques, C. R. Mackay, and D. M. Kaye, "Beyond gut feelings: how the gut microbiota regulates blood pressure," Nature Reviews Cardiology, vol. 15, no. 1, pp. 20-32, 2017.

[77] J. Li, F. Zhao, Y. Wang et al., "Gut microbiota dysbiosis contributes to the development of hypertension," Microbiome, vol. 5, no. 1, p. 14, 2017.

[78] R. Fan, S.-Q. Mao, T.-L. Gu et al., "Preliminary analysis of the association between methylation of the ACE2 promoter and essential hypertension," Molecular Medicine Reports, vol. 15, no. 6, pp. 3905-3911, 2017. 


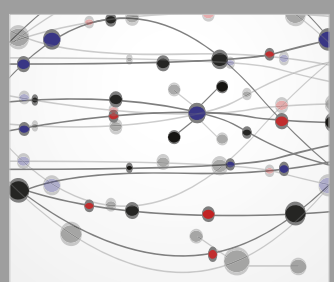

The Scientific World Journal
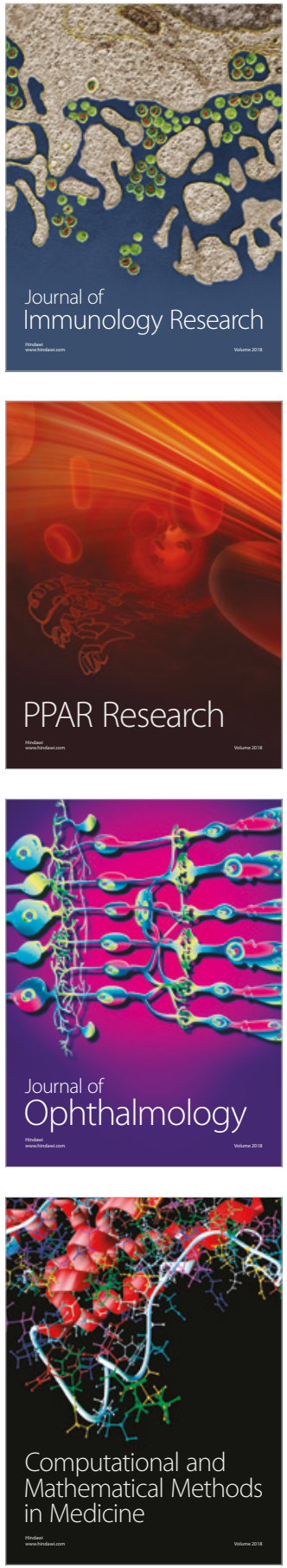

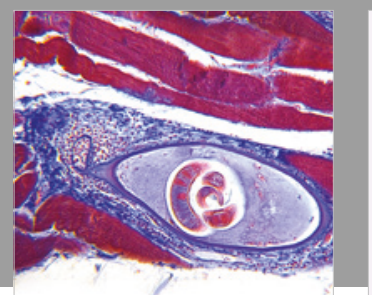

Gastroenterology Research and Practice

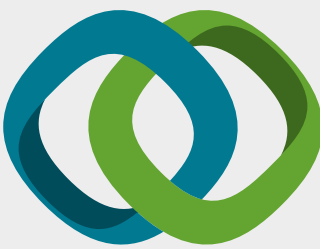

\section{Hindawi}

Submit your manuscripts at

www.hindawi.com
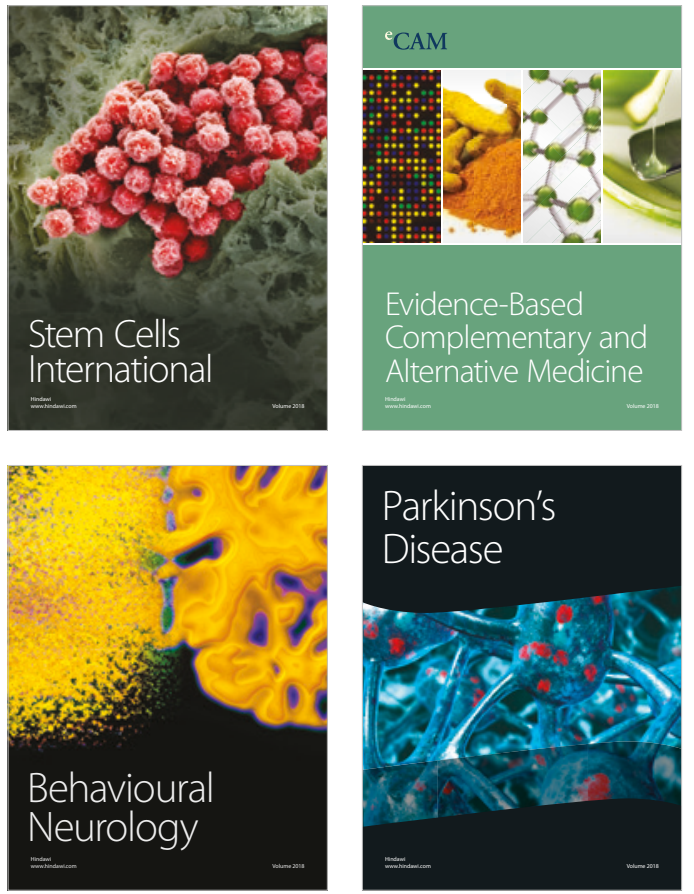

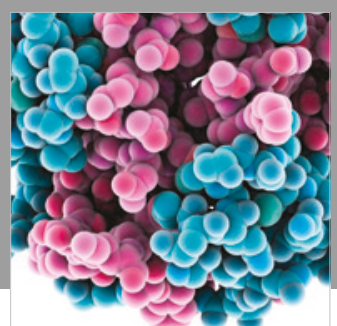

ournal of

Diabetes Research

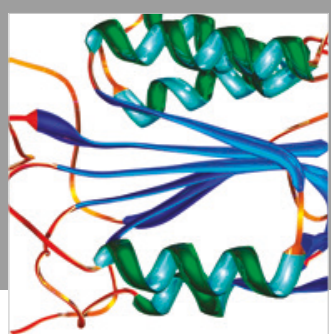

Disease Markers
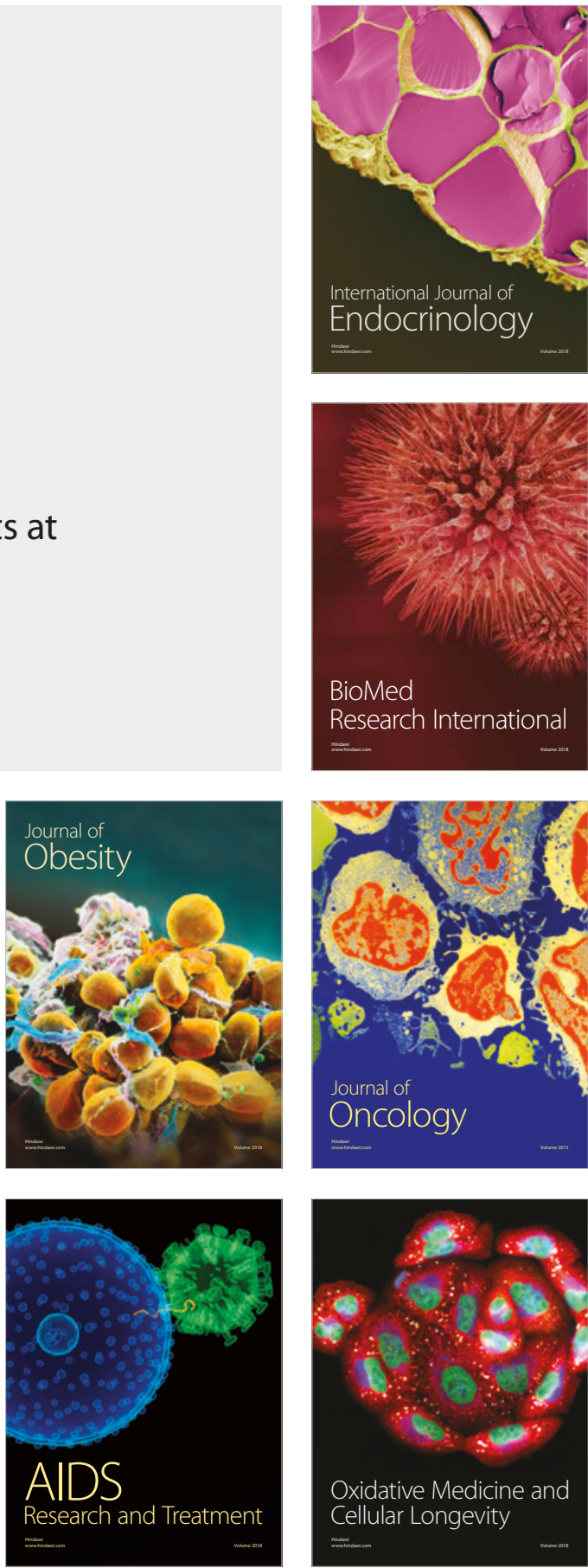